Article

\title{
Integrating Legal and Physical Dimensions of Urban Environments
}

\author{
Ali Aien *, Abbas Rajabifard, Mohsen Kalantari and Davood Shojaei \\ Department of Infrastructure Engineering, The University of Melbourne, Parkville, Melbourne, VIC 3010, \\ Australia; E-Mails: abbas.r@unimelb.edu.au (A.R.); mohsen.kalantari@unimelb.edu.au (M.K.); \\ shojaeid@unimelb.edu.au (D.S.) \\ * Author to whom correspondence should be addressed; E-Mail: aiena@ unimelb.edu.au; \\ Tel.: +61-3-8344-9854; Fax: +61-3-8344-6215.
}

Academic Editor: Wolfgang Kainz

Received: 19 May 2015 / Accepted: 31 July 2015 / Published: 17 August 2015

\begin{abstract}
Building Information Models (e.g., IFC) and virtual 3D city models (e.g., CityGML) are revolutionising the way we manage information about our cities. However, the main focus of these models is on the physical and functional characteristics of urban properties and facilities, which neglects the legal and ownership aspects. In contrast, cadastral data models, such as the Land Administration Domain Model (LADM), have been developed for legal information management purposes and model legal objects such as ownership boundaries without providing correspondence to the object's physical attributes. Integration of legal and physical objects in the virtual 3D city and cadastral models would maximise their utility and flexibility to support different applications that require an integrated resource of both legal and physical information, such as urban space management and land development processes. The aim of this paper is to propose a data model that supports both legal and physical information of urban environments. The methodology to develop this data model is to extend the core cadastral data model and integrate urban features into the data model. The outcome of the research can be utilised to extend the current data models to increases their usability for different applications that require both legal and physical information.
\end{abstract}

Keywords: 3D cadastre; 3D cadastral data model; RRR; 3DCDM; CityGML; LADM; BIM 


\section{Introduction}

\subsection{Background}

The need for more "space" resulting from population growth, urbanisation, and industrialisation has increased the pressure on land-use planning and development. As a result, space above and below ground level is increasingly used. Examples include underground developments, infrastructure facilities, high-rise buildings, and apartments. These three-dimensional (3D) developments not only increase the complexity of the urban environment (physical dimension), but also affect the land and property ownership interests attached to the underlying land (legal dimension).

Virtual 3D city models, such as CityGML, maintain and visualise 3D urban features (3D physical objects) including buildings, ground surface, streets, and vegetation [1]; they do not focus on modelling legal objects. Cadastral data models such as Land Administration Domain Model (LADM) [2] and ePlan [3], on the other hand, manage and maintain the 3D land and property ownership interests, including 3D ownership rights, restrictions, and responsibilities (3D RRRs); they only represent legal objects and do not integrate their physical counterparts. Figure 1 illustrates that cadastral data models defining legal objects of an urban environment, while virtual 3D city models outline its physical objects.

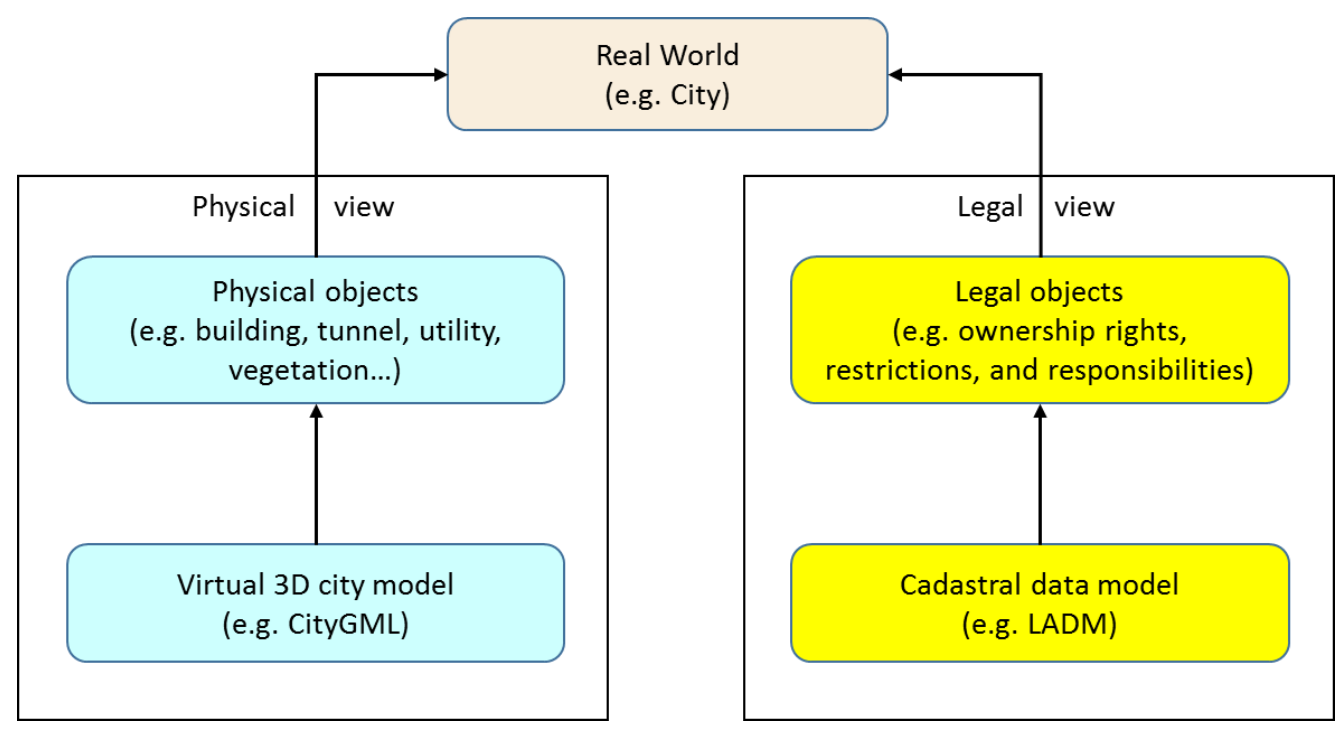

Figure 1. Legal and physical dimensions of an urban environment.

Since ownership boundaries (legal objects) are represented by a building's physical structures such as walls, floors, and ceilings; cadastral data models or virtual 3D city models should maintain and integrate both legal and physical objects of urban features [4]. This integration will maximise the usability of 3D cadastres for additional applications such as property management and city space management.

Integration of legal and physical objects in data models started almost after introduction of CityGML and Building Information model (BIM); however, few researches have been undertaken to investigate extending of cadastral data models to support this integration [4]. The motivation why this integration starts from extending cadastral data models is first existing of strong demand from land authorities to extend their cadastral data models to support 3D cadastre and second availability of legal (cadastral) 
sources such as subdivision plans, titles, and deeds comparing to sources of physical information such BIM and CityGML.

\subsection{Related Work}

Integration of legal and physical objects has been almost started after introduction of CityGML and BIM; however, no substantive research has been done to extent cadastral data models to support physical objects.

An Application Domain Extension (ADE) to CityGML was developed by Dsilva [5] for cadastral purposes. In this ADE, CityGML Building module was extended to represent apartments (condominium units) and some of their legal properties.

El-Mekawy and Östman [6] proposed an extension to unified building model (UBM), which was mainly developed for integrating the building models of both IFC and CityGML standards [7] for dealing with 3D cadastre applications. They investigated shortages of IFC, the most prominent semantic building models for representation of Building Information Model (BIM), CityGML, and UBM that was developed earlier by for modelling complete and real 3D cadastre information system. The result shows that IFC, CityGML, nor the UBM has capabilities for such tasks. They proposed an extension to the UBM and added different subtypes such as "Building Elements Surfaces", "Digging Surfaces", "Protecting Area Surfaces", and "Real Estate Boundary Surfaces" of boundary surfaces to define 3D cadastral information of a building.

Çağdaş [8] also developed an ADE to CityGML to support the requirements of the immovable property taxation system in Turkey. This ADE represents the property unit (i.e., cadastral parcel, condominium unit), their components (i.e., annex, joint facility), and their legal (e.g., property rights) and economic attributes (e.g., tax value, taxation period), as required for the process of property taxation, as well as for cadastral and other administrative processes.

Brinka et al. [9] proposed a new 3D standard for the Netherlands that preserves the 2D data from the existing national standard for large scale topography (Information Model Geography (IMGeo)) and extends them with 3D concepts from CityGML. CityGML-IMGeo contains generic aspects that can be reused for extending other domain information models such as for 3D cadastre.

Gozdz et al. [10] investigated possibilities of applying CityGML for cadastral purposes by introducing a CityGML-LADM ADE model that provides relations between spatial objects from legal and physical world. Ying et al. [11] introduced a framework and a workflow of the conversion from CityGML to 3D cadastral. El-Mekawy et al. [12] described problems and solutions concerning interaction between BIM and the registration and visualisation of 3D real property information.

Isikdag et al. [13] explored the possible role of semantically rich 3D building models and 3D cadastres in relation to valuation and taxation.

Soon et al. [14] proposed a semantics-based fusion framework to integrate CityGML and 3D LandXML. Rönsdorf et al. [15] suggested a modelling solution to integration some parts of LADM and CityGML.

The Open Geospatial Consortium has proposed InfraGML (successor of LandXML), a GML application schema supporting land development and civil engineering infrastructure facilities. Anticipated subject areas include alignment, road, rail, survey, terrain, land parcels, drainage, wastewater, 
and water distribution systems. The focus of InfraGML is to cover the content of LandXML and additionally support modern survey equipment [16].

\subsection{Problem Description}

Since ownership boundaries are defined based on the actual structures of a development (e.g., building) in cadastres, the location of boundaries must be clear. Therefore, visualising ownership boundaries alone without integrating with their physical counterparts still will not solve existing ambiguities that affect interpretation of the relationship between the rights and building interests, and will not reduce boundary confusion among owners and owner corporations. Integration of physical and legal objects, by contrast, will clearly represent the ownership rights and restrictions [4].

Virtual 3D city models, such as CityGML, do not support objects to represent detailed information required to represent legal ownership boundaries and their physical structure counterparts [6]. Cadastral data models such as LADM also do not maintain and represent physical counterparts of legal objects [4].

\subsection{Methodology}

So far all challenges to integrate cadastral legal objects with their physical counterparts have been focused on extending virtual 3D city models to support cadastral objects. In this paper, however, the core cadastral data model is extended to support the corresponding physical objects.

This paper proposes a 3D cadastral data model ( $3 D C D M)$ as a solution capable of supporting 3D data, integrating 3D physical objects with their corresponding 3D legal objects, and featuring semanticallyenriched objects. The data model is developed based on the ISO standards and UML modelling language is used to specify the data model. The 3DCDM model represents 3D legal objects and connects legal and physical objects together. In this regard, the $3 D C D M$ model is equipped with the concepts of the Legal Property Object (LPO) and the Physical Property Object (PPO). The first facilitates modelling of all existing interests (RRR) as legal objects. The second considers all 3D urban features such as buildings, tunnels, and utilities as physical objects. 3D geometric primitives of GML (Geographic Markup Language) such as Solid and MultiSurface are used to define the Legal and Physical Property Objects. The 3DCDM model supports semantics that define every aspect of legal and physical objects and, therefore, it facilitates their integration. In the 3DCDM's UML diagrams, physical hierarchy is represented in light blue and legal hierarchy is represented in yellow to be easily distinguishable. Root classes are in light brown, Geometry classes are in green, and Address in purple. The 3DCDM's class names are written in Italic format and its abstract classes are hyphened such as "_AbstractClass".

\subsection{Scope and Delimitation}

Since the main contribution of this paper is to represent the $3 D C D M$ model in detail, which integrates legal and physical objects, assessment of virtual 3D city models and cadastral data models in terms of supporting integration of legal and physical objects is beyond the scope of this paper, which has been investigated previously by El-Mekawy and Östman [6] and Aien et al. [4]. Attributes of the 3DCDM's classes are not also described in this paper because of space limitations. 
The remainder of the paper presents the model in detail. Section 2 describes the core component of the 3DCDM. Section 3 illustrates all classes, associations, and attributes of the 3DCDM. The 3DCDM is evaluated by the project's stakeholders in Section 4. A brief discussion on the processing of changes in the data model is described in Section 5. Research conclusions are presented in Section 6.

\section{Conceptual Model of the 3DCDM}

The methodology to develop the conceptual data model of the $3 D C D M$ is to develop the conceptual model from the core cadastral data model step by step. In the first step, the conceptual model of the core cadastral data model is represented. Then, this data model is modified to support the concept of the Legal Property Object. The logical model of the Legal Property Object model is created and its components are demonstrated. The Legal Property Object model forms the legal hierarchy of the 3DCDM model. The Physical hierarchy of the $3 D C D M$ model that supports the physical counterparts of the legal objects (Legal Property Objects) maintains related objects such as building, tunnel, utility, and terrain model. Both hierarchies are described in the following sections.

\subsection{Conceptual Model of the Core Cadastral Data Model}

Cadastres are dealing with entities consisting of interests in land that have three main components: Parcel, Right, and Person [17,18]. The spatial unit of a legal object (Right) are surveyed and demarcated by geometrical objects, while their legal descriptions (legal documents and interest holder information) are kept in the land registry (Figure 2).

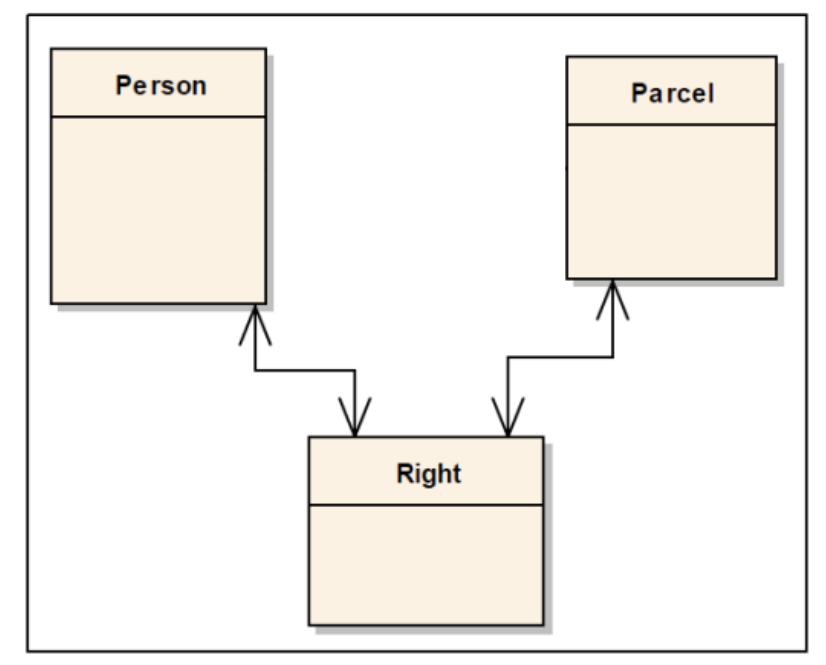

Figure 2. Main components of current cadastral data models.

The laws define the outlines of the legal objects. The legal objects normally are described by boundaries that demarcate where a right or a restriction ends and where the next begins and the contents of that right [19].

The UML diagram in Figure 3 represents the core components of most current cadastral systems [2,3,20-22]. In this model, Parcel and Right are linked together by unique identifiers (the parcel identifier). Parcel identifier is employed for organising land information. 
Since cadastres are now under pressure from various land-related commodities and stratified, spatial unit (parcel)-based indexing of interests in land cannot accommodate interests that are not necessarily equivalent to the extent of spatial units or land parcels [23]. Legal Property Object model restructures the concept of the core cadastral data model from a land parcel-based data model to a spatially referenced data model.

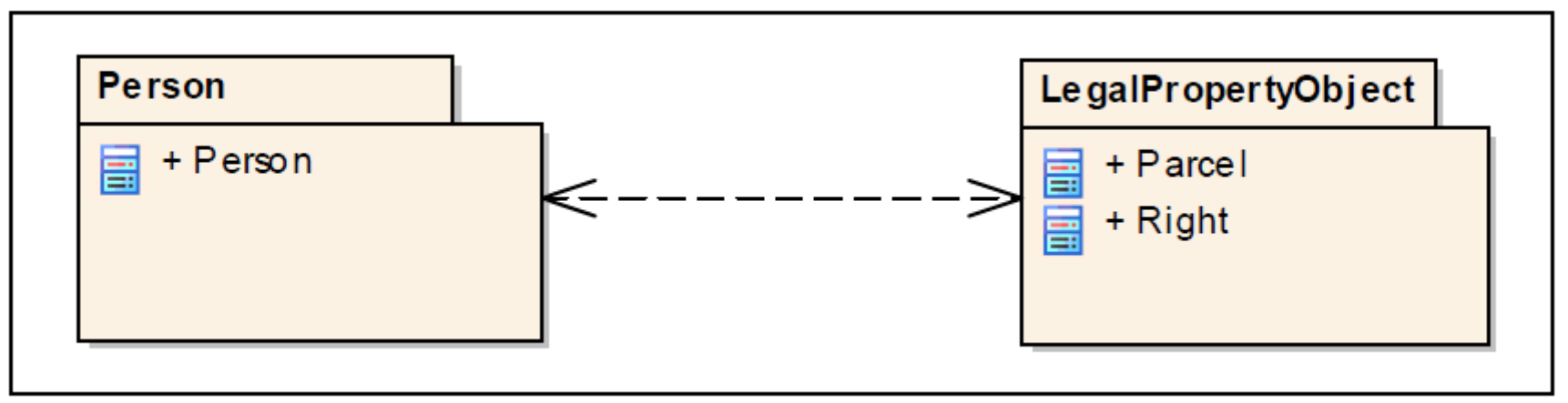

Figure 3. New cadastral data model based on the Legal Property Object concept, adopted from [23].

\subsection{Conceptual Data Model of the Legal Property Object}

The Legal Property Object was proposed by Kalantari [23] to replace the core cadastral data model by a spatially-referenced data model based on the Legal Property Object concept that uniquely combines every interest and its spatial extent. The Legal Property Object includes a particular interest with its spatial dimensions.

Although land interests (RRRs) can be maintained by different organisations and have particular characteristics that drive RRRs as a separate class in cadastral data models [24], combining RRRs and SpatialUnit classes into one class, Legal Property Object class (Figure 3), facilitates the incorporation of a wide range of RRRs into the cadastral system. Interoperability and simplicity in data-exchange processes, particularly upgrading and updating cadastral databases using spatial referencing systems, are promoted [25].

Once the advantages of utilising the concept of Legal Property Object have been described, utilisation of this concept in the $3 D C D M$ model can be considered. The following discussion describes how the Legal Property Object model is used in the $3 D C D M$ model.

\subsection{Utilisation of the Legal Property Object (LPO) Model in the 3DCDM}

The 3DCDM model is developed based on the concept of the Legal Property Object. The Legal Property Object allows for the representation of spatial aspects of legal objects (2D and 3D). The UML diagram of Legal Property Object model is depicted in Figure 4. In this model, the LegalPropertyObject represents all land interests (rights, restrictions, and responsibilities). It is created using its associated features such as LegalDocument, InterestHolder, Geometry and Address. 


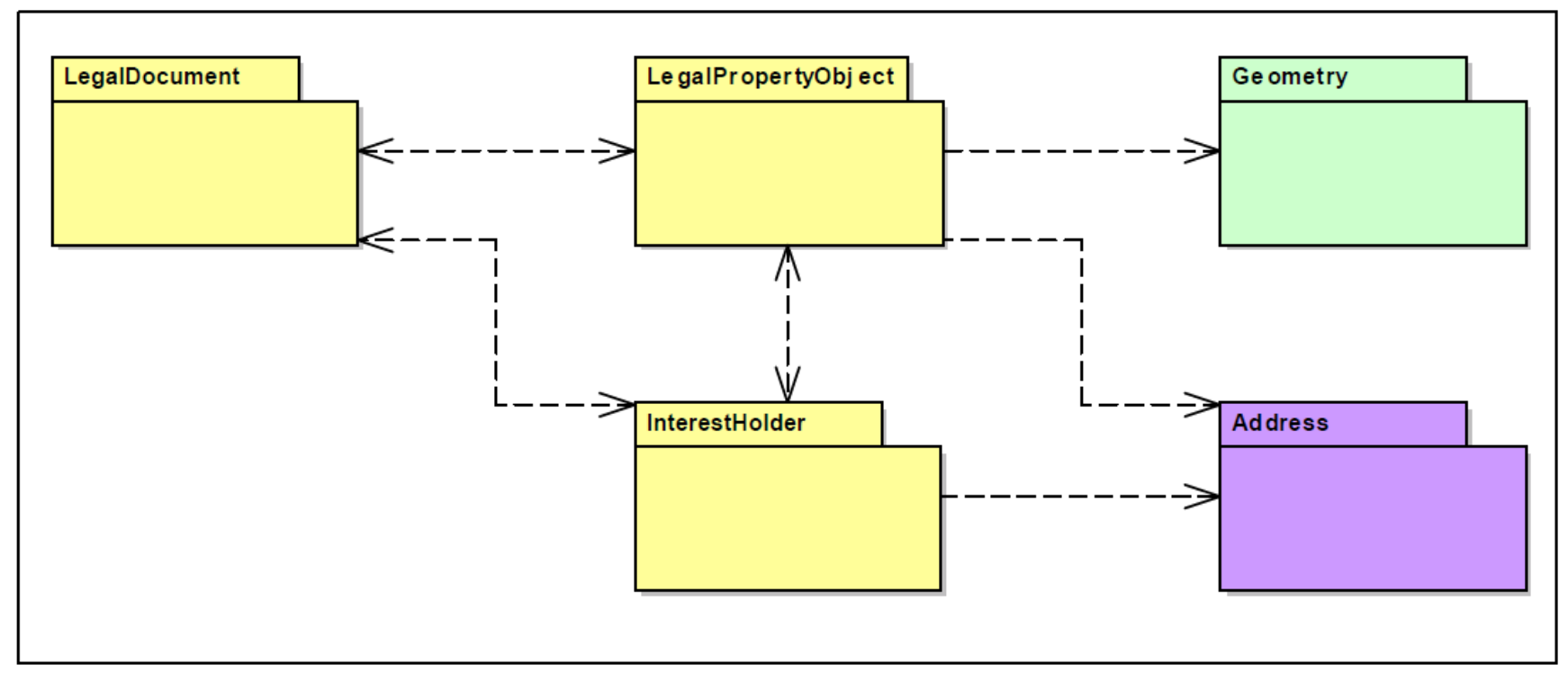

Figure 4. LegalPropertyObject's package view, adopted from [25].

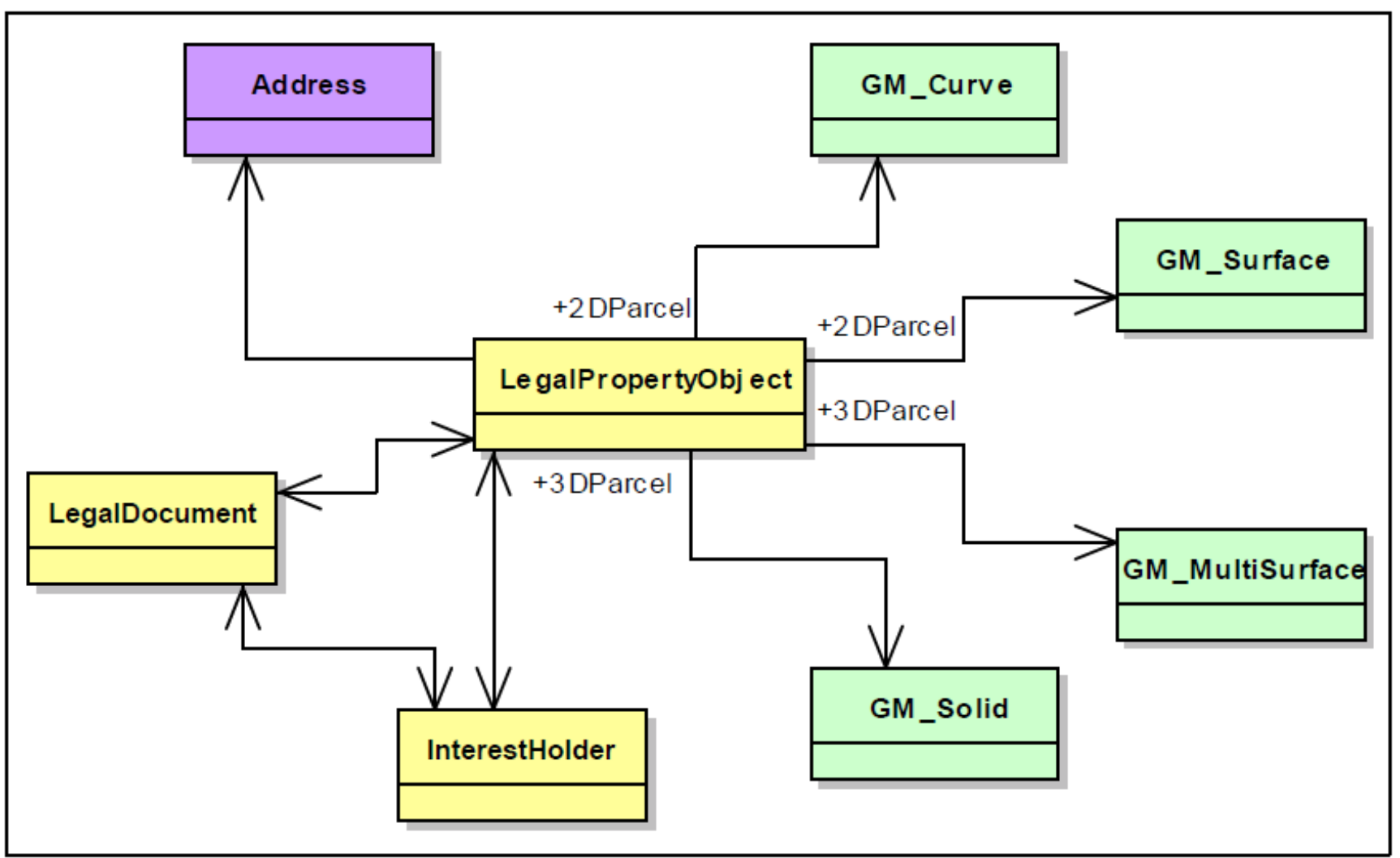

Figure 5. Geometric representation of the LegalPropertyObject.

The LegalDocument package contains authoritative and registered documents such as titles, deeds, or agreements. It defines rights or ownership of property attached to the Legal Property Object. The InterestHolder package maintains information about the interest holder of the Legal Property Object and the Address package maintains the physical address of the Legal Property Object and living address of the interest holder. The Geometry package includes zero, one, two, and three-dimensional geometric 
primitives to represent spatial extent of the Legal Property Object. The Legal Property Object model is the core component of the $3 D C D M$ model.

\subsection{Geometry of LegalPropertyObject Class in the 3DCDM}

In The 3DCDM model, LegalPropertyObject class plays the role of a 3D parcel. It contains required legal object information and its spatial properties are represented by $2 \mathrm{D}$ or $3 \mathrm{D}$ geometrical primitives [26].

The geometric representation of a LegalPropertyObject class is shown in Figure 5. If the LegalPropertyObject is a 2D object such as a land parcel, it can be represented by $1 \mathrm{D}$ geometric primitives, such as GM_MultiCurve or with GM_MultiSurface, and if it is a 3D object, such as a volumetric space (3D parcel), a 3D geometric primitives, such as GM_Solid, is used.

Few cadastral data models support 3D parcels to model 3D RRRs. However, they do not use solid geometry (Solid) to represent 3D parcels. Solid geometry facilitates 3D representation, volumetric calculation, and 3D spatial analysis. The 3DCDM model supports solid representation [4].

\subsection{Integration of Legal and Physical Objects in the 3DCDM Model}

There is a need for integration between legal objects and their physical counterparts for 3D cadastral applications [4]. Boundaries of 3D parcels (ownership spaces) are defined based on the actual structures of a building to determine the legal volume. In the paper based plans of subdivision, the boundaries of ownership are only representative. For building subdivisions, from legal perspective, the actual structure of the building defines the ownership spaces. In other words, the legal definition is equal to the physical definition. In the digital world it is, therefore, necessary to have a physical definition of the buildings in the cadastres. Therefore, visualising 3D parcels only in 3D cadastres does not clearly represent the actual information of ownership rights, restriction, and responsibilities. Using 3D parcels alone without integrating with their physical counterparts, such as building structures, still will not solve existing ambiguities that affect interpretation of the relationship between the rights and building interests, and will not reduce boundary confusion among owners and owner corporations. Integration of physical and legal objects, by contrast, will clearly represent the legal structure of a building. Figure 6 illustrates how physical information (_PhysicalPropertyObject) is associated with the LegalPropertyObject with the purpose of having more realistic 3D objects in 3D cadastres.

The LegalPropertyObject represents any type of legal object. Each LegalPropertyObject has one or more owners (InterestHolder). Each LegalPropertyObject is associated with zero or more abstract class _PhysicalPropertyObject, which has four subclass specialisations: Building, Land, Tunnel, _UtilityNetwork, and PhysicalPropertyObject (any type of Physical Property Object such as a mine, road, or sea). The association between LegalPropertyObject and_PhysicalPropertyObject is bi-directional, which means that it is possible to navigate from each hierarchy (legal or Physical) to find the corresponding object.

Abstract class_PhysicalPropertyObject is a superclass in the physical hierarchy of the $3 D C D M$ model, while class LegalPropertyObject is the core class in the legal hierarchy.

Physical Property Objects are important for cadastral applications. Having separate class PhysicalPropertyObject facilitates a selection of an appropriate PhysicalPropertyObject for any 
particular application. In terms of modelling a building, only Building subclass is used. Also class Building can be aggregated with class Tunnel and_UtilityNetwork's subclasses to provide more comprehensive model. Each subclass has its own subclasses and different types of interrelationships between feature classes like aggregations, generalisations, and associations.

In the 3DCDM model, all physical and legal objects have a unique objectID in the entire model. In this method, in the legal hierarchy, a LegalPropertyObject is generated and an objectID is assigned to that. Also, in the physical hierarchy, a PhysicalPropertyObject such as a building, a unit, a wall, or a water network is created and its corresponding legal object is referenced by attributes (objectID). If the PhysicalPropertyObject and its counterpart have a similar geometry (for example, when a wall is an interior boundary of ownership right), then the geometry is only created for the PhysicalPropertyObject and the geometry of the corresponding LegalPropertyObject will be represented by referencing to its physical object.

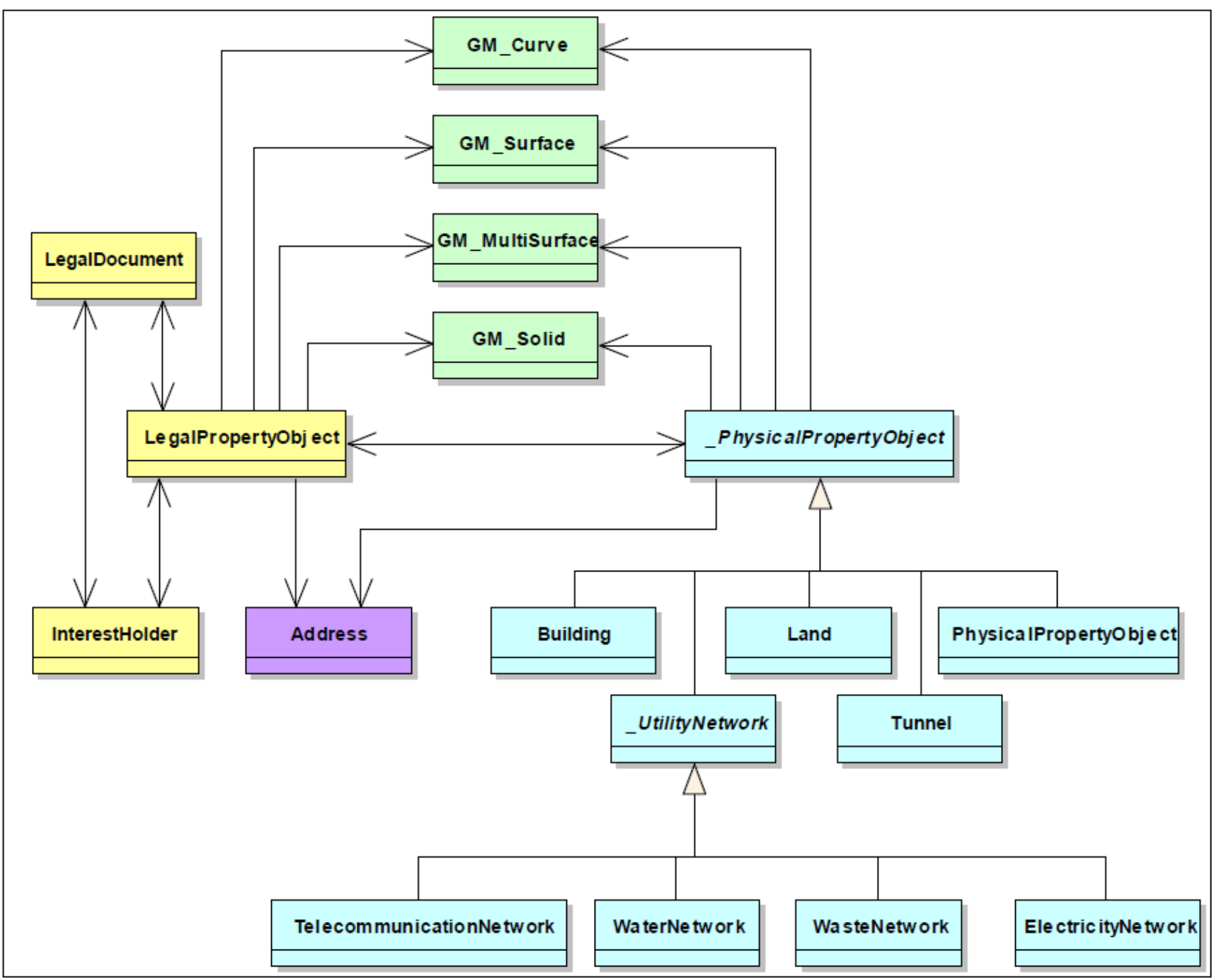

Figure 6. Conceptual model of integrating the legal and physical objects in the 3DCDM model. 


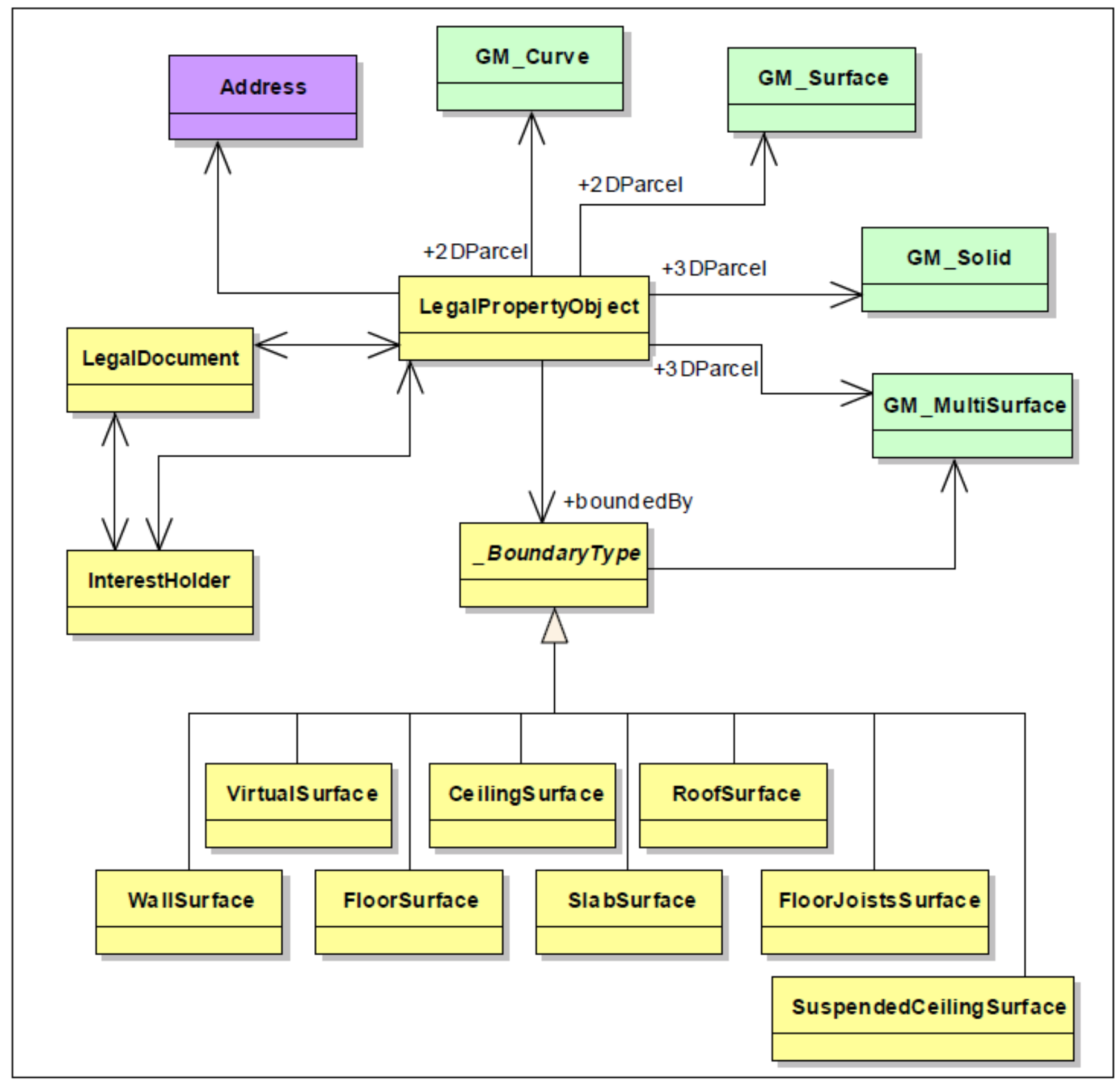

Figure 7. Spatially and semantically coherent conceptual model of the LegalPropertyObject.

\subsection{Utilisation of Semantics in the 3DCDM Model}

There is a need to utilise semantics in cadastral models. Therefore, these models can be used for visualisation, thematic queries, and spatial analysis purposes.

The 3DCDM model integrates the legal and physical objects with the semantics associated with the data. There is a coherent modelling of semantics and geometrical properties in the 3DCDM model. Furthermore, attributes and associations between features support semantics.

Semantically, legal objects are represented by the LegalPropertyObjects. LegalPropertyObject's attributes identify the type of the legal object and further support semantics. For example, the LegalPropertyObject object can be an ownership, common property, easement, covenant, or 
license. Physical objects also are represented by features such as Building, Land, Tunnel, and_UtilityNetwork (Figure 6).

Spatially, geometrical objects represent the location, boundary and extent of the legal and physical objects, while each geometrical object is instantiated by semantics in the 3DCDM model. Figure 7 represents how geometrical objects (green objects) are linked to their corresponding semantics.

In this model, solid geometry (GM_Solid) is used to spatially create a 3D LegalPropertyObject, such as a volumetric ownership space of a unit in a building. In addition, BoundayType class specialisations such as VirtualSurface, WallSurface, FloorSurface, and CeilingSurface are used to semantically enrich the created LegalPropertyObject. This concept applies to the physical objects as well. Therefore, all legal and physical objects in the $3 D C D M$ model are enriched with semantics.

\subsection{Components of the 3DCDM Model}

Surveying information including observations and cadastral control points, which are important elements in cadastral plans, are considered as a separate module in the 3DCDM model. Figure 8 represents the conceptual model of the $3 D C D M$, which includes the main features of the 3D cadastre. As previously discussed, the 3DCDM model has two hierarchies, legal and physical. Survey and CadastralPoint are included in the legal hierarchy. They cover the required surveying information of the Legal Property Object.

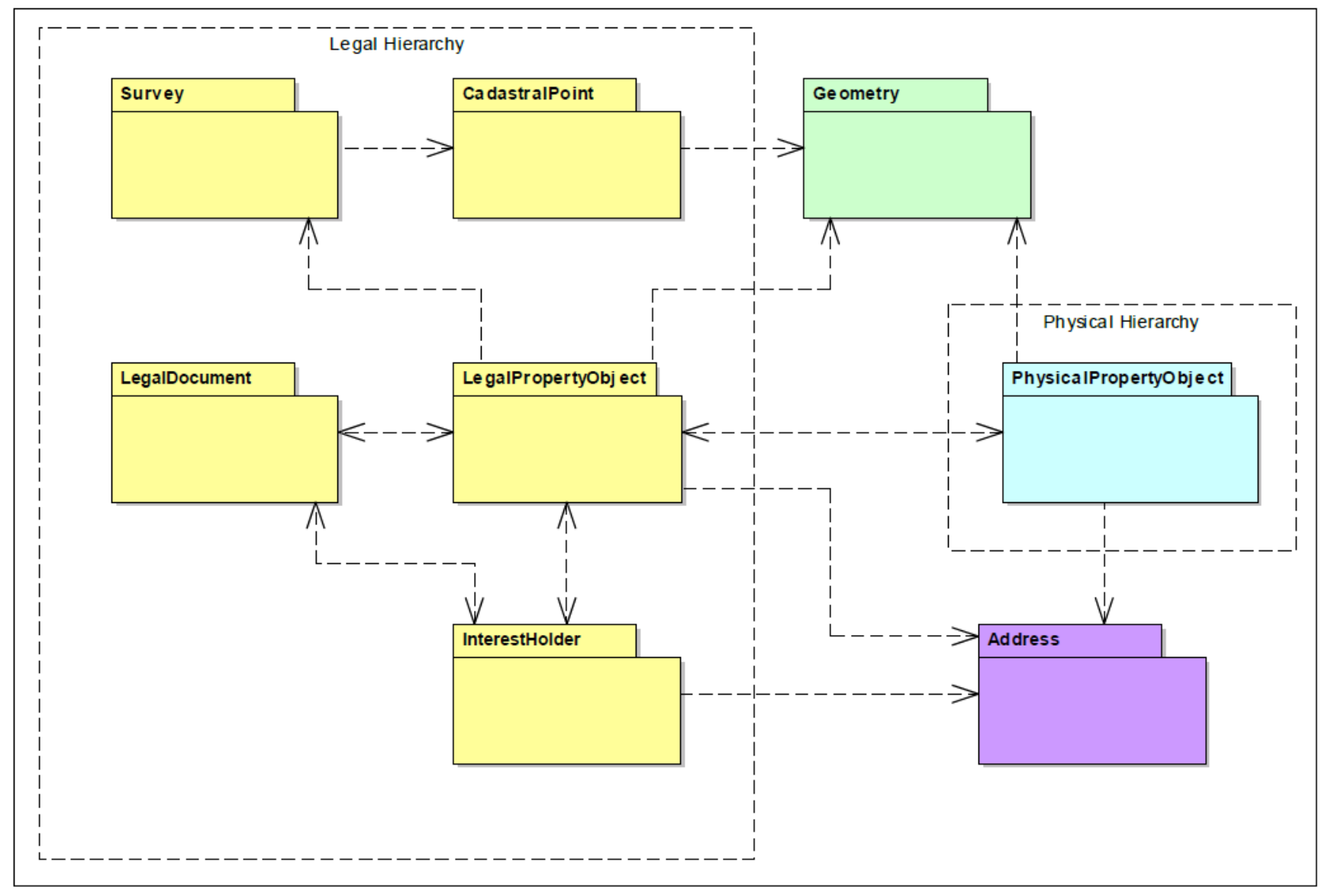

Figure 8. Conceptual Model of the 3DCDM. 


\section{Logical Model of the 3DCDM}

In this section, the logical data model of the $3 D C D M$ model is presented based on the conceptual model of the $3 D C D M$ model. The $3 D C D M$ model is implemented as an application schema of the Geography Markup Language 3 (GML3), version GML3.2.1, the extendable international standard for spatial data exchange and encoding issued by the Open Geospatial Consortium (OGC) and the ISO TC211. The 3DCDM model inherits GML3's specifications. GML3.2.1 supports most of the 3DCDM's general and spatial requirements. GML 3.2.1 maintains wide variety of spatial reference systems; it has a rich geometric model and provides essential attributes to develop the 3DCDM model (Figure 9). Different components and classes of the $3 D C D M$ model are represented in this section in detail.

\subsection{DCDM Hierarchies and Components}

The 3DCDM model is composed of two hierarchies: legal and physical. However, they are connected to each other through the associations between their subclasses. The 3DCDM's users can navigate through each hierarchy independently and also between hierarchies.

Each hierarchy consists of different components and they are all connected to the core component of the $3 D C D M$ model, which is called the root model. The root model contains the basic features on the 3DCDM model. The root model must be implemented in any conformant system.

The legal hierarchy of the 3DCDM model comprises of the following components: LegalPropertyObject, Survey, CadastralPoint, and InterestHolder. The physical hierarchy has the following components: PhysicalPropertyObject, Building, Land, Tunnel, UtilityNetwork, and Terrain. The 3DCDM model supports the combination of different legal and physical components to provide more comprehensive cadastral model (Figure 9).

The geometry model of the $3 D C D M$ is based on the geometry model of Geographic Markup Language (GML3.2.1). GML follows the specification of standard ISO 19107 "Spatial schema" [27]. GML represents 3D geometry according to the well-known Boundary Representation [28]. There is a geometrical primitive for each dimension: Point for a zero-dimensional object, Curve for a one-dimensional, Surface for two-dimensional objects, and Solid for three-dimensional objects. A solid is bounded by surfaces and a surface by curves [29].

Combined geometries can be aggregates, complexes or composites of primitives. For an aggregate, the spatial relationship between primitives is not defined. They may be disjoint, touching, or overlapping. GML provides a special aggregate for each dimension, a MultiPoint, a MultiCurve, a MultiSurface, and a MultiSolid. On the contrary, a complex is topologically structured. Complex elements must be disjointed and must not overlap. They must be topologically connected along their boundaries. A composite is a special complex that can only contain elements of the same dimension. A composite can be a CompositeCurve, CompositeSurface, and a CompositeSolid [27].

The Geometry model contains the various classes for coordinate geometry [27]. All of these classes, through the root class, inherit an optional association to a coordinate reference system. The geometry package has several internal packages that separate primitive geometric objects, aggregates, and complexes, which have a more elaborate internal structure than simple aggregates [29]. Figure 10 show one part of the $3 D C D M$ geometry model. 


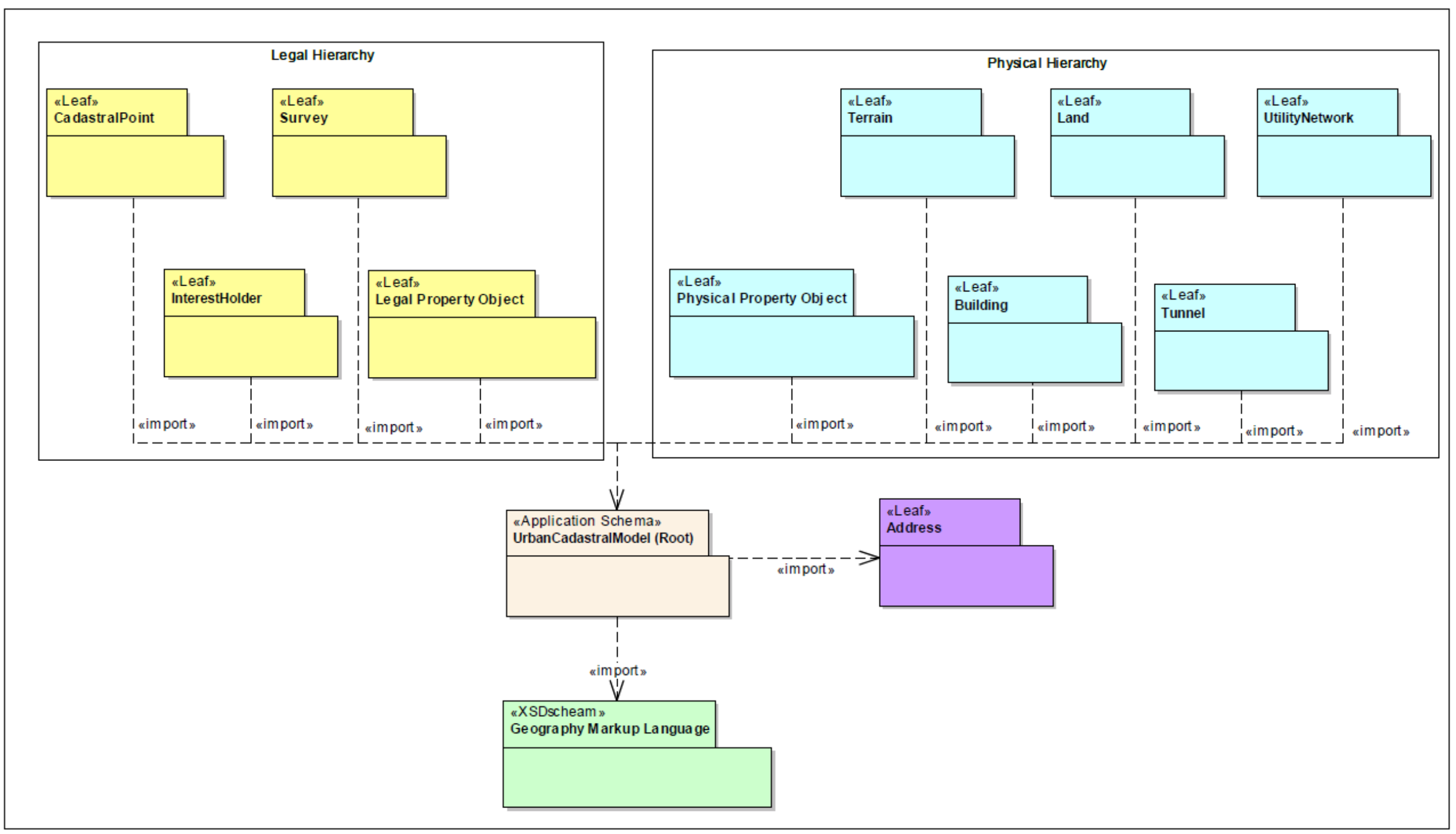

Figure 9. Separate models and hierarchies of the 3DCDM model. 


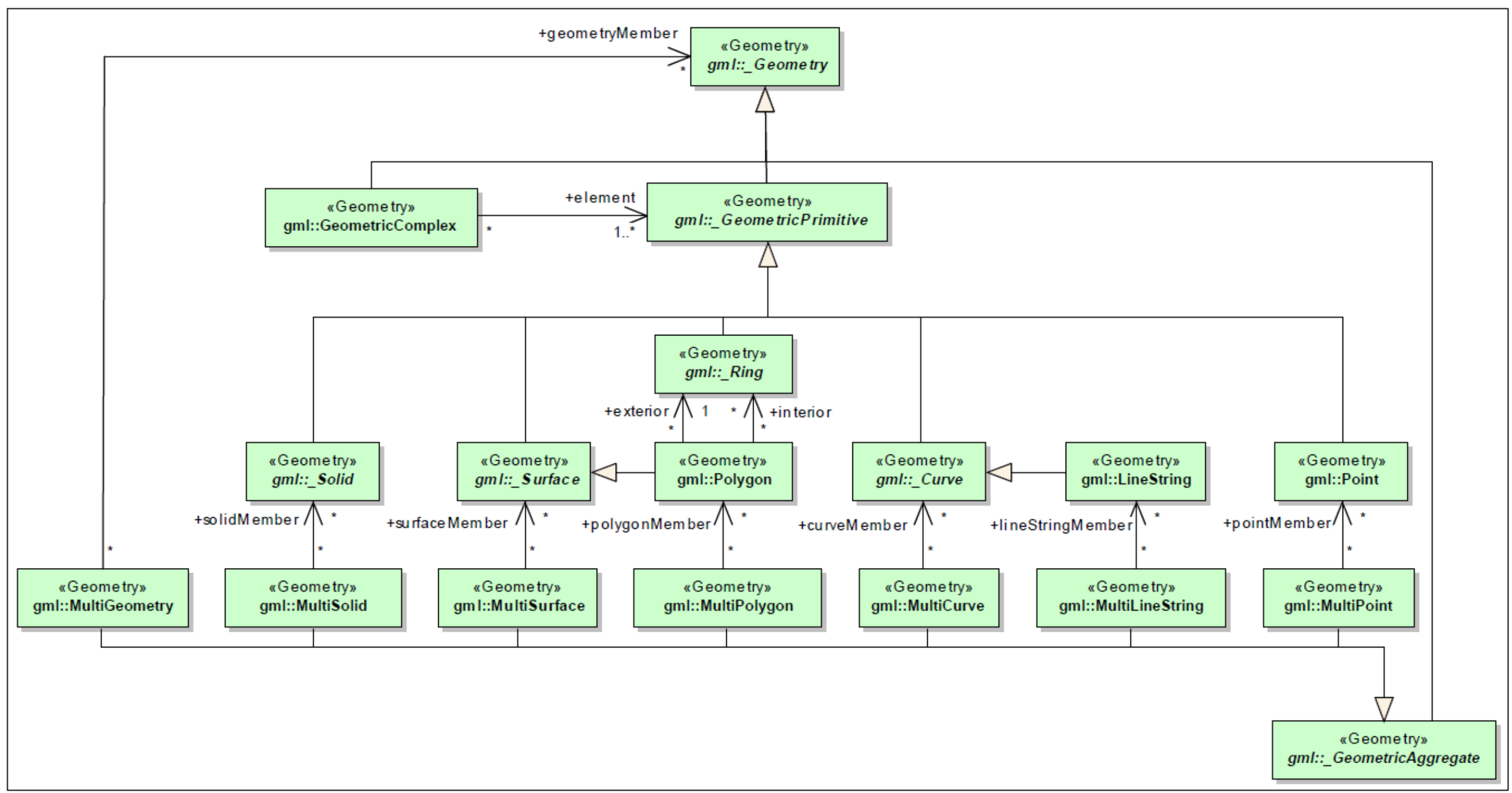

Figure 10. A part of 3DCDM Geometry Model—(profile of GML3.2.1). 


\subsection{DCDM Root Model}

The 3DCDM Root model comprises of core features and components of the 3DCDM model. All other models are connected to the root. This component of the $3 D C D M$ model must be realised in the implementation phase of the data model. The root model consists of two legal and physical hierarchies and it provides the opportunity for the user to choose one of or both hierarchies. The UML diagram in Figure 11 illustrates 3DCDM's root model.

The 3DCDM's root model consists of gml:_Feature, gml;_FeatureCollection, UrbanCadastralModel, Application, MericUnit, CadastralModel,_CadastralObject, UrbanModel, and_UrbanObject.

\section{gml:_Feature}

This abstract element serves as the head of a substitution group, which may contain any elements. Abstract gml:_Feature may be thought of as "anything that is a GML feature" [29].

gml:_FeatureCollection

Abstract class gml:_FeatureCollection is a subclass of the GML class gml:_Feature and is a collection of properties that apply to the gml:_FeatureCollection and an optional list of Spatial Reference System Definitions.

\section{UrbanCadastralModel}

UrbanCadastralModel is the base class of all components in the 3DCDM model. Its adminArea attribute enables specification of the area where the cadastral model is created. UrbanCadastralModel is a subclass of the GML class gml:_Feature. UrbanCadastralModel consists of zero or more CadastralModel and UrbanModel (Figure 11). Therefore, it inherits the metadata property such as Coordinate Reference System (CRS) and general properties such as name from its superclass.

Application

This class provides further metadata about the application that created the model. MetricUnit.

This class provides further metadata about the measurement units.

CadastralModel

This class is the first class of the 3DCDM's legal hierarchy. It forms the cadastral model and contains the legal objects.

_CadastralObject

Abstract class_CadastralObject is the base class of all legal classes in the 3DCDM. It is a subclass of gml:_Feature._CadastralObject subclasses are LegalPropertyObject, InterestHolder, Survey, and CadastralPoints (Figure 12). 


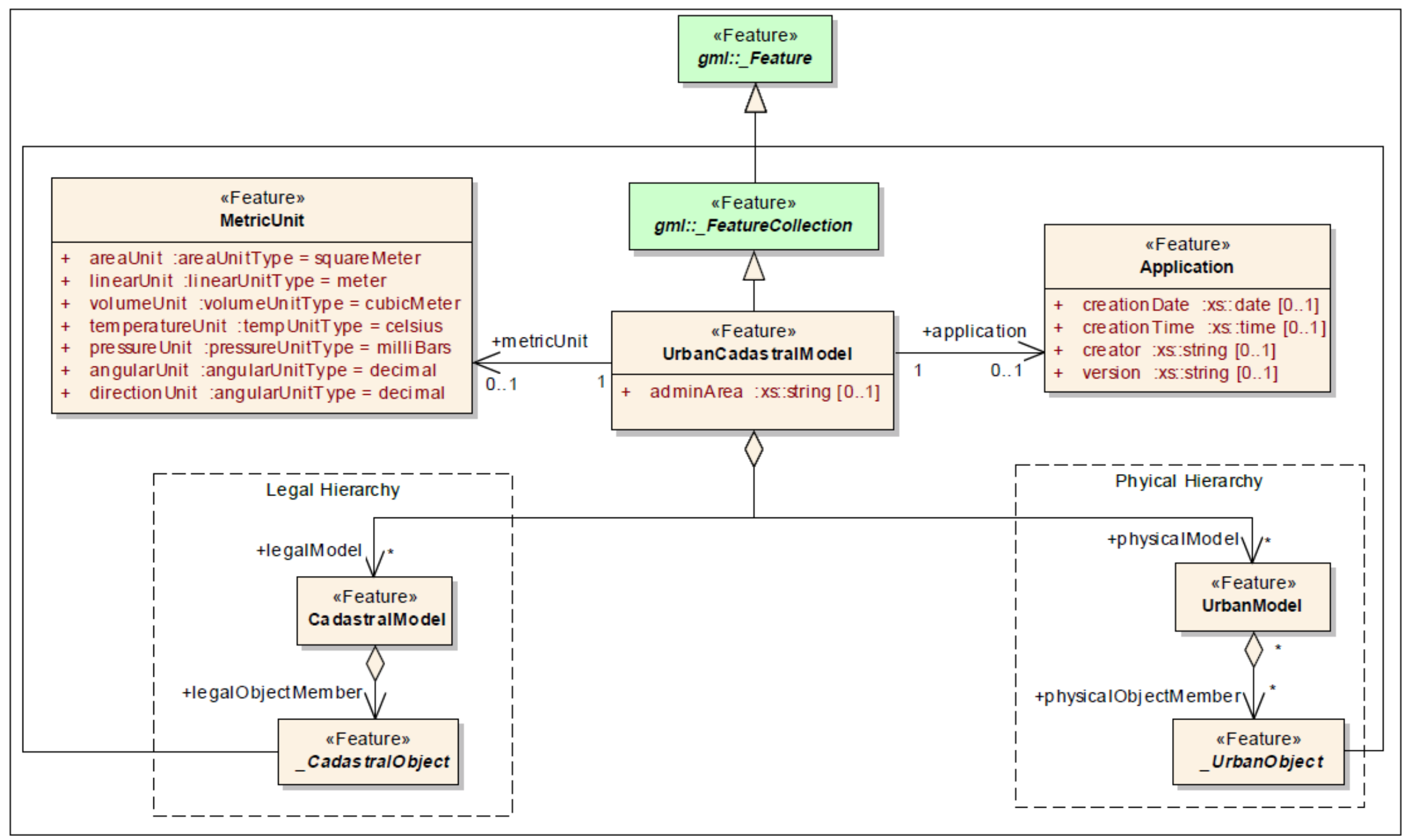

Figure 11. UML diagram of the 3DCDM Root model. 


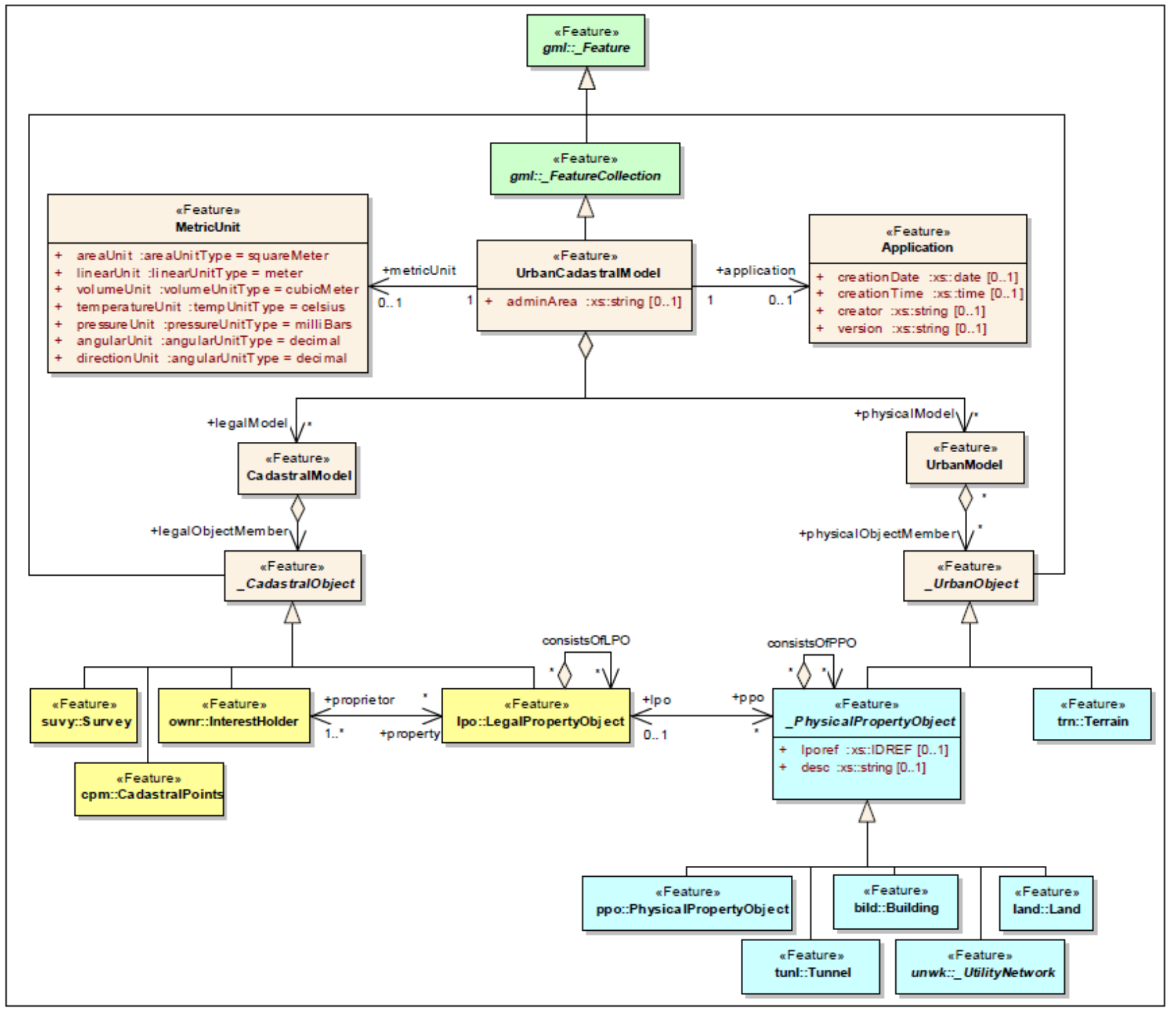

Figure 12. The UML diagram of 3DCDM Root model and subclasses of the legal and physical models.

\section{UrbanModel}

This class is the first class of the $3 D C D M$ 's physical hierarchy. It forms the urban model and contains physical objects.

\section{_UrbanObject}

Abstract class_UrbanObject is the base class of all physical classes in the 3DCDM. It is a subclass of gml:_Feature._UrbanObject subclasses are _PhysicalPropertyObject, Terrain, Building, Land, Tunnel,_UtilityNetwork, and_PhysicalPropertyObject(Figure 12).

\subsection{DCDM LegalPropertyObject Model}

Every legal object is represented as a LegalPropertyObject in the 3DCDM model. Figure 13 represents the attributes and associated classes of the class LegalPropertyObject. Each LegalPropertyObject 
class is associated with zero or more_PhysicalPropertyObjects, such as Building, Tunnel, _UtilityNetwork, and Land. It has one or more InterestHolders. Each LegalPropertyObject acquires its legal information through its associated LegalDocument.

Ownership space is more of a conceptual concept. Physical structures such as fences, hedges, or walls are used to demarcate and represent the ownership boundaries. However, the legal spaces (boundaries) are not always equal to their corresponding physical spaces (physical boundaries). For example, legal boundaries (ownership volumes) are usually bigger than the physical boundaries (building) in villa units. Furthermore, in some cases such as airspace, the legal space cannot be defined by physical boundaries. For these reasons, the creation of Legal Property Object in 3D cadastres is needed. The 3DCDM supports creation of LegalPropertyObject using GML geometry objects. If the LegalPropertyObject is a 2D land parcel, it can be created using gml:MultiCurve or gml:MultiSurface. And if it is a $3 \mathrm{D}$ parcel, it can be created using gml:MultiSurface or gml:_Solid (Figure 14).

However, if the LegalPropertyObject is equal to its physical counterpart and can be defined using physical objects; the 3DCDM provides various boundary types such as WallSurface, CeilingSurface, FloorSurface, RoofSurface, SlabSurface, SuspendedCeilingSurface, or FloorJoistSurface to semantically define the boundary of a LegalPropertyObject.

Another method to semantically define the legal boundaries in the $3 D C D M$ is to use the LegalPropertyObject's attribute ppoRef to reference a physical object.

LegalPropertyObject

This class represents all types of legal objects such land parcel, 3D parcel, ownership, easement, and common property. LegalPropertObject is a subclass of _CadastralObject. LegalPropertObject is associated to the class Address that identifies the physical address of the LegalPropertObject.

LegalDocument: Title

Each LegalPropertyObject is associated with one legal document such as a title, a deed, an agreement if it has been registered. It is also possible to identify the LegalPropertyObject through the legal documents (Figure 13).

LegalDocument: ParentTitle

Each Title can be associated to zero or more ParentTitle (Figure 13).

LegalDocument: LandDescription

LegalPropertyObject's specification is described in class LegalDescription (Figure 13).

LegalDocument: Mortgage

Title can be associated to the class Mortgage (Figure 13). Mortgage is registered as a restriction in the Title. 


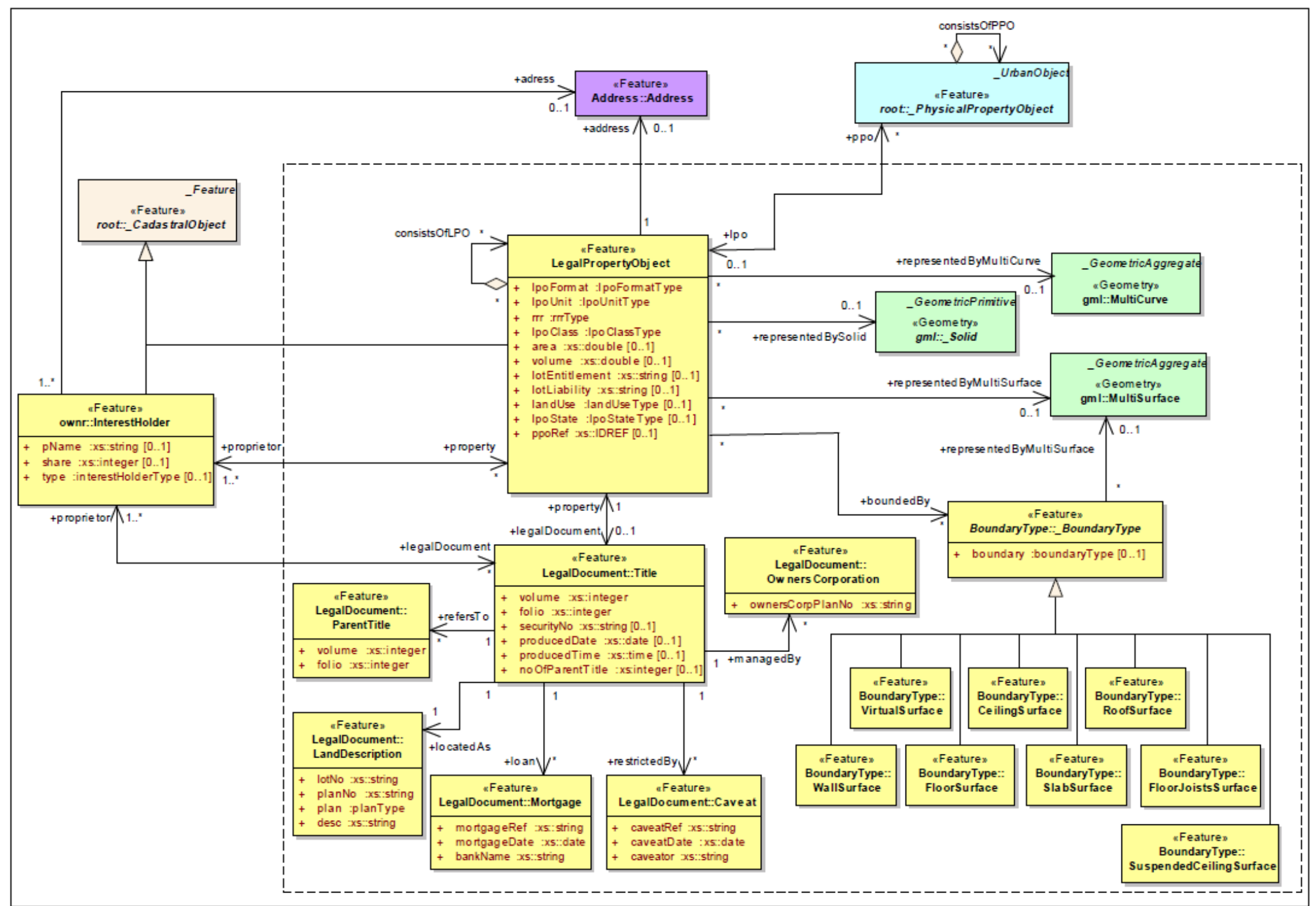

Figure 13. The UML diagram of 3DCDM LegalPropertyObject model. 


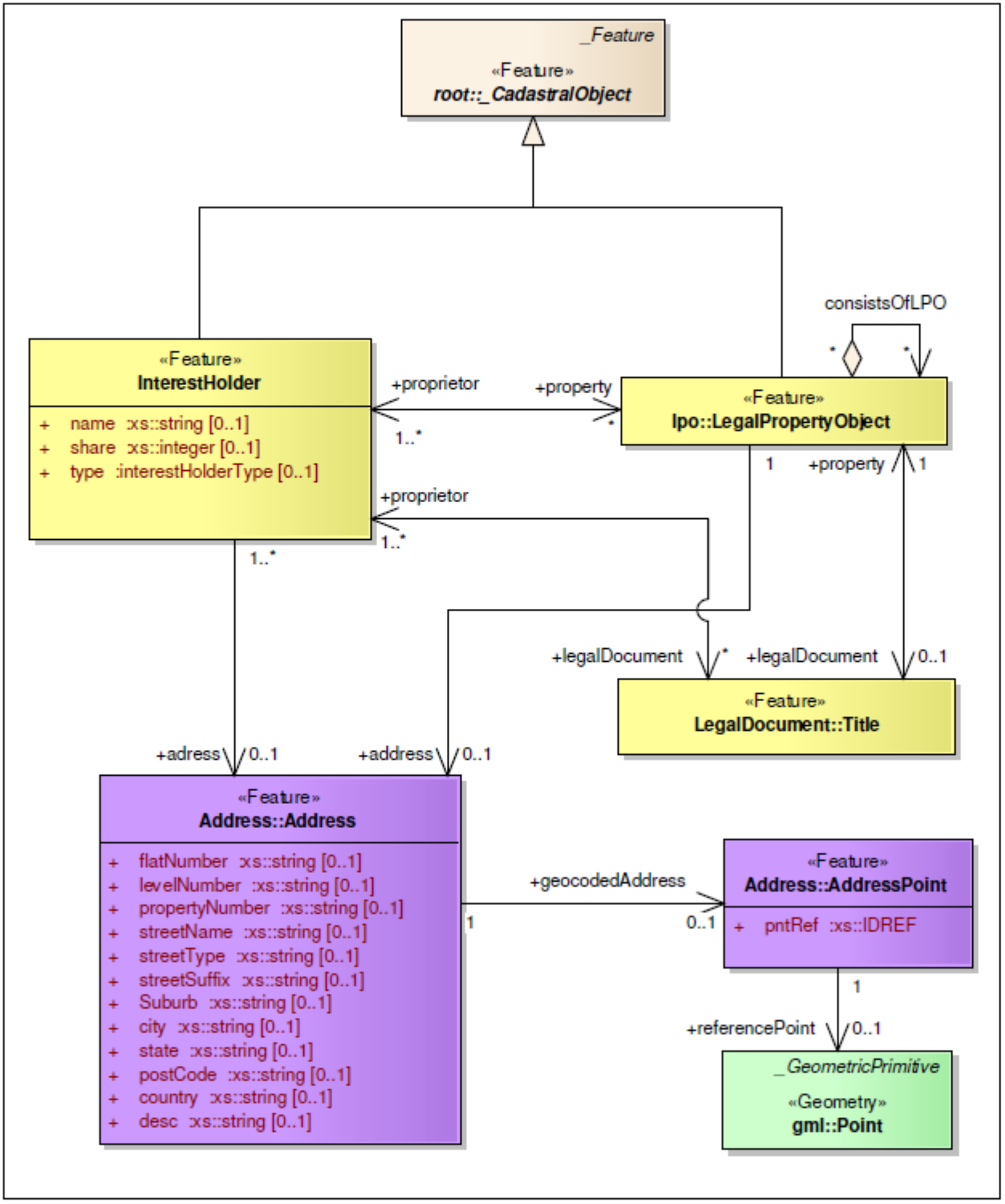

Figure 14. The UML diagram of 3DCDM InterestHolder model.

LegalDocument: Caveat

Title can be associated to the class Caveat (Figure 13). Caveat is registered as a restriction in the Title. 
LegalDocument: OwnersCorporation

Title can be associated to the class OwnersCorporation (Figure 13). OwnersCorporation is registered to manage the property when it has common areas.

BoundaryType:_BoundaryType

Abstract class _BoundaryType contains different subclasses to semantically define the LegalPropertyObject. They are VirtualSurface, WallSurface, CeilingSurface, FloorSurface, RoofSurface, SlabSurface, SuspendedCeilingSurface, and FloorJoistsSurface (Figure 13).

\subsection{DCDM InterestHolder Model}

This model maintains information about the land interest holder. This model has only one class, which is class InterestHolder.

InterestHolder

InterestHolder can be a person, group, or an organisation that have certain rights (RRRs) over a particular 3D space. An InterestHolder can be associated with the classes LegalPropertyObject and Title. InterestHolder is a subclass of _CadastralObject (Figure 14). InterestHolder is associated to the class Address, which identifies the living address of the interest holder.

\subsection{DCDM Survey Model}

The Survey model contains seven classes that describe technical and administrative information of surveying and surveyor, which is required in cadastral applications.

Survey

The class Survey is the main class of the model and holds general information of surveying (Figure 15). Survey is a subclass of_CadastralObject.

Surveyor

The class Surveyor maintains the details of any surveyor who participated in the survey (Figure 15).

SetupInstrument

The class SetupInstrument retains setting-up information of surveying instruments on surveying points (Figure 15).

SetupPoint

The class SetupPoint refers to the setup points of the cadastral points (Figure 15). 


\section{ObservationGroup}

Surveying observations are maintained in the class ObservationGroup and its associated classes RedHorizontalArcObservation and RedHorizontalLineObservation (Figure 15).

\section{RedHorizontalArcObservation}

The class RedHorizontalArcObservation holds horizontally reduced observations for arcs (Figure 15).

\section{RedHorizontalLineObservation}

The class RedHorizontalLineObservation holds horizontally reduced observations for lines (Figure 15).

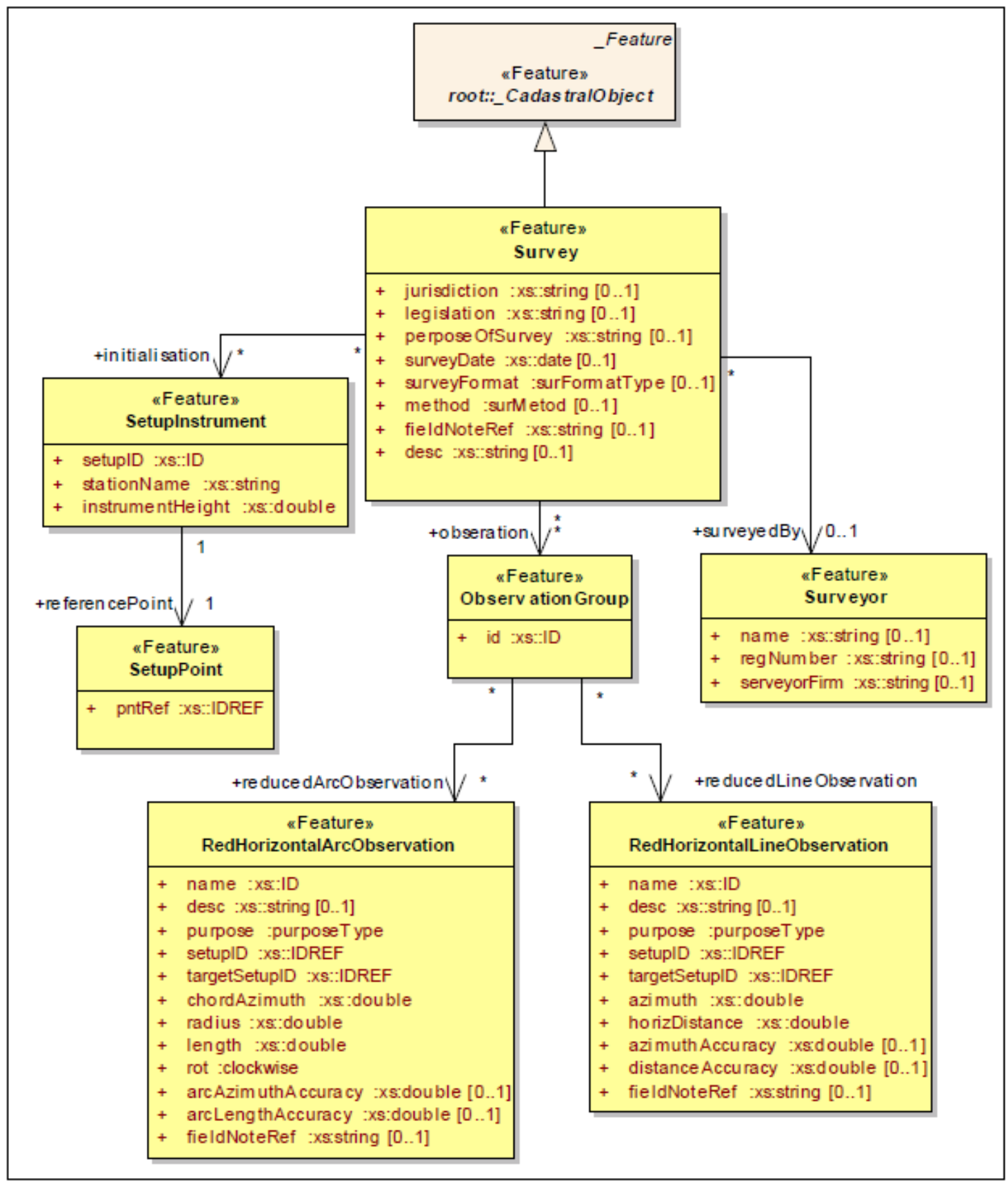

Figure 15. The UML diagram of 3DCDM Survey model. 


\subsection{DCDM CadastralPoints Model}

As surveying of permanent marks is very important for surveyors, the $3 D C D M$ model keeps the elements related to the survey permanent marks as the CadastralPoints model. Permanent marks are placed in the ground by monuments (e.g., pin, peg) during the survey process. Survey points can be 2D or 3D. The CadastralPoints model contains two main classes that describe the information of survey points (Figure 16).

CadastralPoints

The class CadastralPoints is a subclass of_CadastralObject. The class CadastralPoints is a group of survey points (Figure 16).

CadastralPoint

The class CadastralPoints consists of one or more CadastralPoint, which is created using gml:point geometry object (Figure 16).

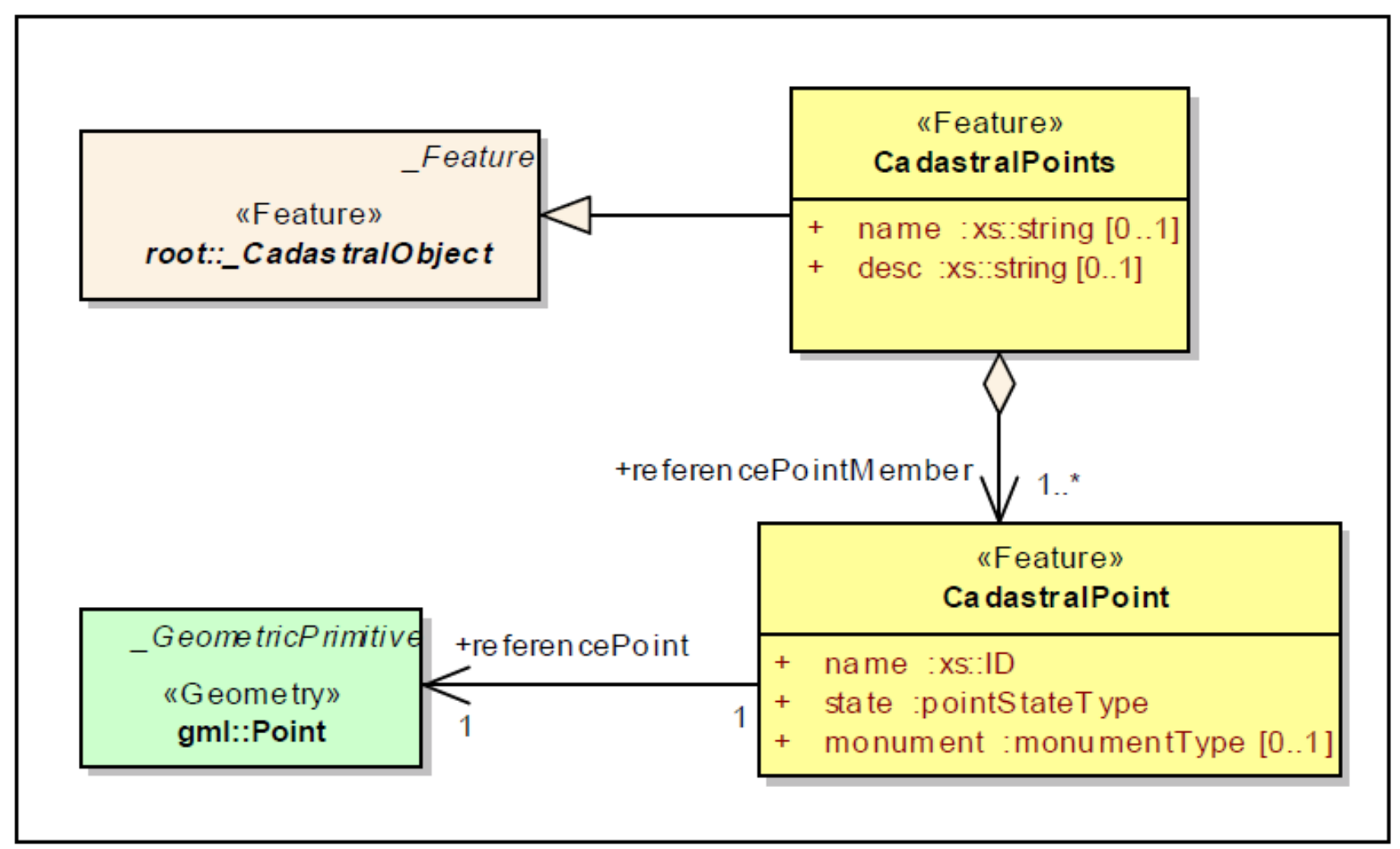

Figure 16. The UML diagram of 3DCDM CadastralPoints model.

\subsection{DCDM Building Model}

The 3DCDM supports the Building model. The 3DCDM model allows users to create the building models and then link it to its legal counterparts. The Building model is located in the physical hierarchy of the 3DCDM model. The Building model has an abstract superclass_PhysicalPropertyObject that is associated with zero or one LegalPropertyObject. The abstract class_PhysicalPropertyObject is a 
subclass of _UrbanObject. The Building model can be created through four main subclasses. They are class Building, abstract class_BuildingPart, class Space, and abstract class _StructuralComponent (Figure 17).

Building

The class Building describes the physical shape (without internal components) of a building. The class Building is a 3D object and is created using gml:_Solid or gml:MultiSurface geometry object. The class Building's set of attributes help to almost envisage the shape of the building. In the 3DCDM model, the intersection lines of a building and terrain surface is represented using gml:MultiCurve. The class Building is associated to the class Address to specify the physical address of each building. A building is decomposed into different building parts (Figure 17).

_BuildingPart

The abstract class_BuildingPart and its specialisations such as Unit, CarPark, Roof, Pathway, ServiceRoom, StorageRoom, Balcony, and BuildingPart (any type of building part) are used to model the internal sections of a building (Figure 17).

Space

Each building part can be created using zero or more spaces. The class Space defines the internal volumetric space of a_BuildingPart. Space is a 3D object and can be represented using gml:MultiSurface or gml:_Solid (Figure 17).

_StructuralComponent

Each building part can be created using zero or more_StructuralComponent. The abstract class _StructuralComponent defines the structures that are used to create the _BuildingPart. Each _StructuralComponent can be represented using gml:MultiSurface or gml:_Solid. The abstract class _StructuralComponent's semantically defines the type of building structure (Figure 17). They are Wall, Ceiling, Floor, Door, Window, and Structure (any type of structure such as a pile).

\subsection{DCDM Land Model}

Land model in the 3DCDM model is used for 2D cadastral applications. In this model, it considers a physical object that legal entities will be attached to. The Land model has an abstract superclass _PhysicalPropertyObject that is associated with zero or one LegalPropertyObject.

Land

The class Land is a subclass of abstract class_PhysicalPropertyObject. The class Land is a 2D object and is created using gml:MultiCurve or gml:MultiSurface geometry object. The class Land is associated to the class Address to specify the physical address of each land (Figure 18). 


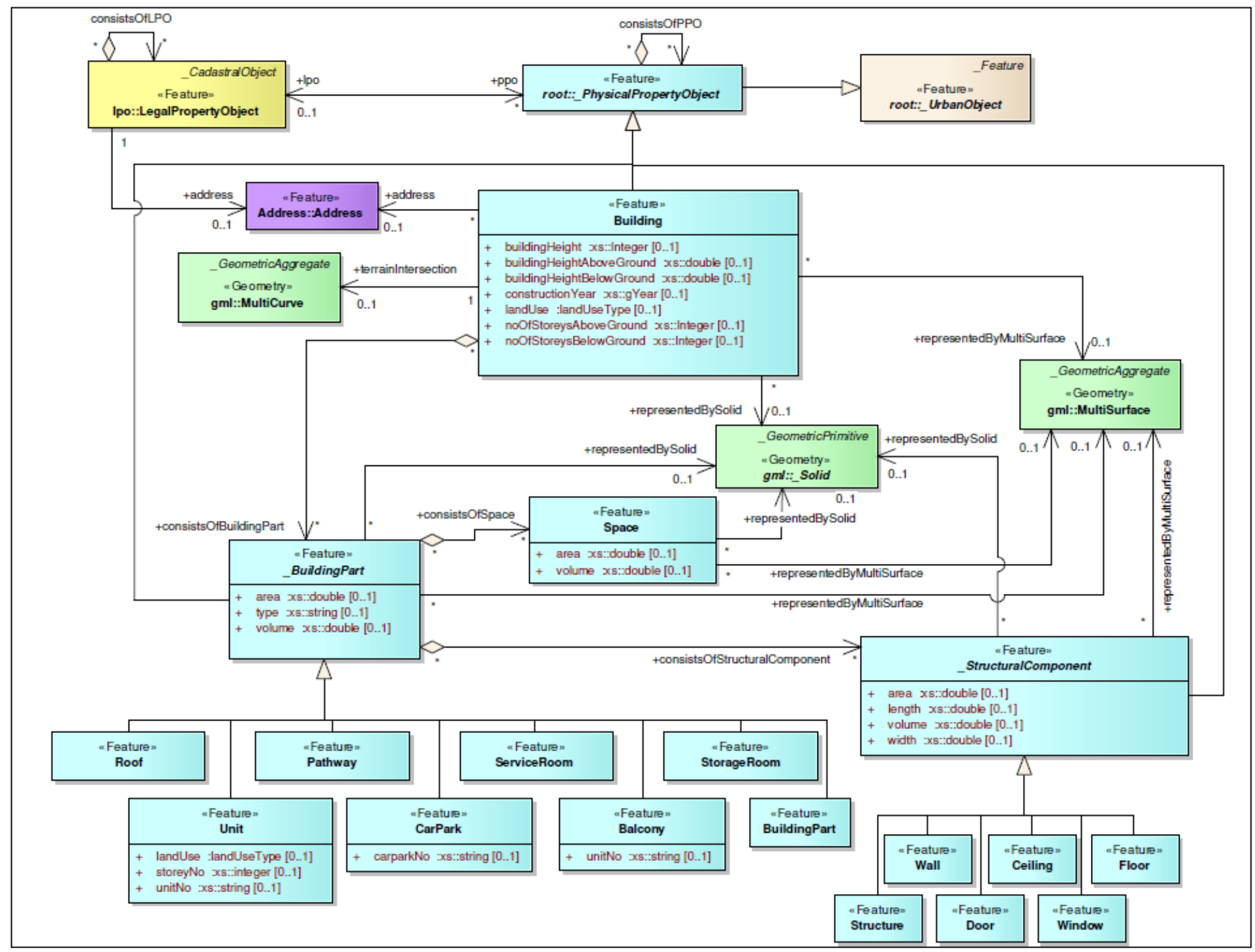

Figure 17. The UML diagram of 3DCDM Building model. 


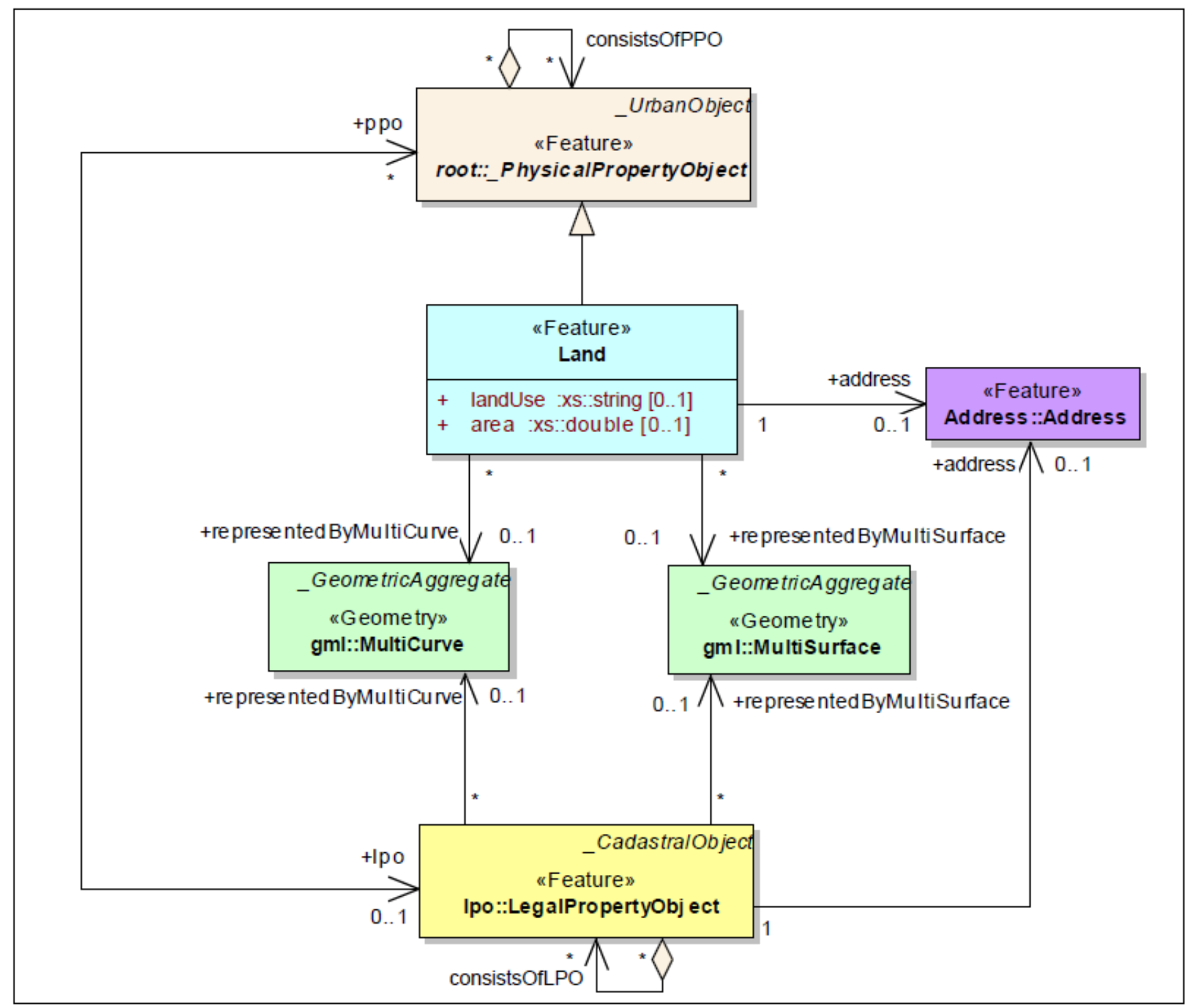

Figure 18. The UML diagram of 3DCDM Land model.

\subsection{DCDM Tunnel Model}

The 3DCDM model allows users to create the tunnel models and then link it to its legal counterparts. The Tunnel model, such as Building and Land models, is located in the physical hierarchy of the 3DCDM model. The Tunnel model has an abstract superclass_PhysicalPropertyObject, which is associated with zero or one LegalPropertyObject. The Tunnel model can be created through three main subclasses. They are class Tunnel, class Space, and abstract class_StructuralComponent (Figure 19). A tunnel is not always an underground object and it could be a walkway that connects buildings above street.

Tunnel

The class Tunnel describes the physical shape (without internal components) of a tunnel. The class Tunnel is a 3D object and is created using gml:_Solid or gml:MultiSurface geometry object. In the $3 D C D M$ model, the intersection lines of a tunnel and terrain surfaces is represented using 
gml:MultiCurve. The class Tunnel is associated to the class Address to specify the physical address of a tunnel (Figure 19).

Space

Each tunnel can be created using zero or more spaces. The class Space defines the internal volumetric space of a Tunnel. Space is a 3D object and can be represented using gml:MultiSurface or gml:_Solid (Figure 19). Attributes of the class Space in Tunnel model are similar to attributes of the class Space in the Building model.

\section{_StructuralComponent}

Each tunnel can be created using zero or more _StructuralComponent. The abstract class _StructuralComponent in the Tunnel model is similar to the abstract class_StructuralComponent in Building model. Figure 20 illustrates how a tunnel consists of structures and a space.

\subsection{DCDM UtilityNetwork Model}

The $3 D C D M$ model allows users to create the utility network models and then link it to its legal counterparts. The UtilityNetwork model can be created through two main subclasses. They are abstract class_UtilityNetwork and class NetworkComponent (Figure 21).

\section{_UtilityNetwork}

The abstract class_UtilityNetwork and its specialisations such as ElectricityNetwork, WaterNetwork, TelecommunicationNetwork, and WasteNetwork are used to model the internal sections of a utility network. The abstract class _UtilityNetwork is associated to the class Address to specify the physical address of a utility network (Figure 21).

Each_UtilityNetwork can be represented directly using GML geometric objects or can be represented by various network components.

\section{NetworkComponent}

Each utility network can be created using zero or more NetworkComponent. The class NetworkComponent defines the structures that are used to create the _UtilityNetwork. Each NetworkComponent can be represented using gml:MultiCurve, gml:MultiSurface, or gml:_Solid (Figure 21).

\subsection{DCDM PhysicalPropertyObject Model}

In the $3 D C D M$ model, the PhysicalPropertyObject is designed to model unknown urban objects, which need to be created to link to their corresponding legal objects. If the physical property object were none of the existing physical models in the 3DCDM (it is not a Building, Land, Tunnel, nor _UtilityNetwork models), PhysicalProperty_Object model would be used to model this urban object. The PhysicalPropertyObject model has an abstract superclass_PhysicalPropertyObject that is associated 
with zero or one LegalPropertyObject. The PhysicalPropertyObject model has one subclass that is class PhysicalPropertyObject (Figure 22).

PhysicalPropertyObject

The class PhysicalPropertyObject is used to model any unknown urban object that is not included in the model. Each PhysicalPropertyObject can be represented directly using GML geometric objects. The class PhysicalPropertyObject is associated to the class Address to specify the physical address of an urban object (Figure 22).

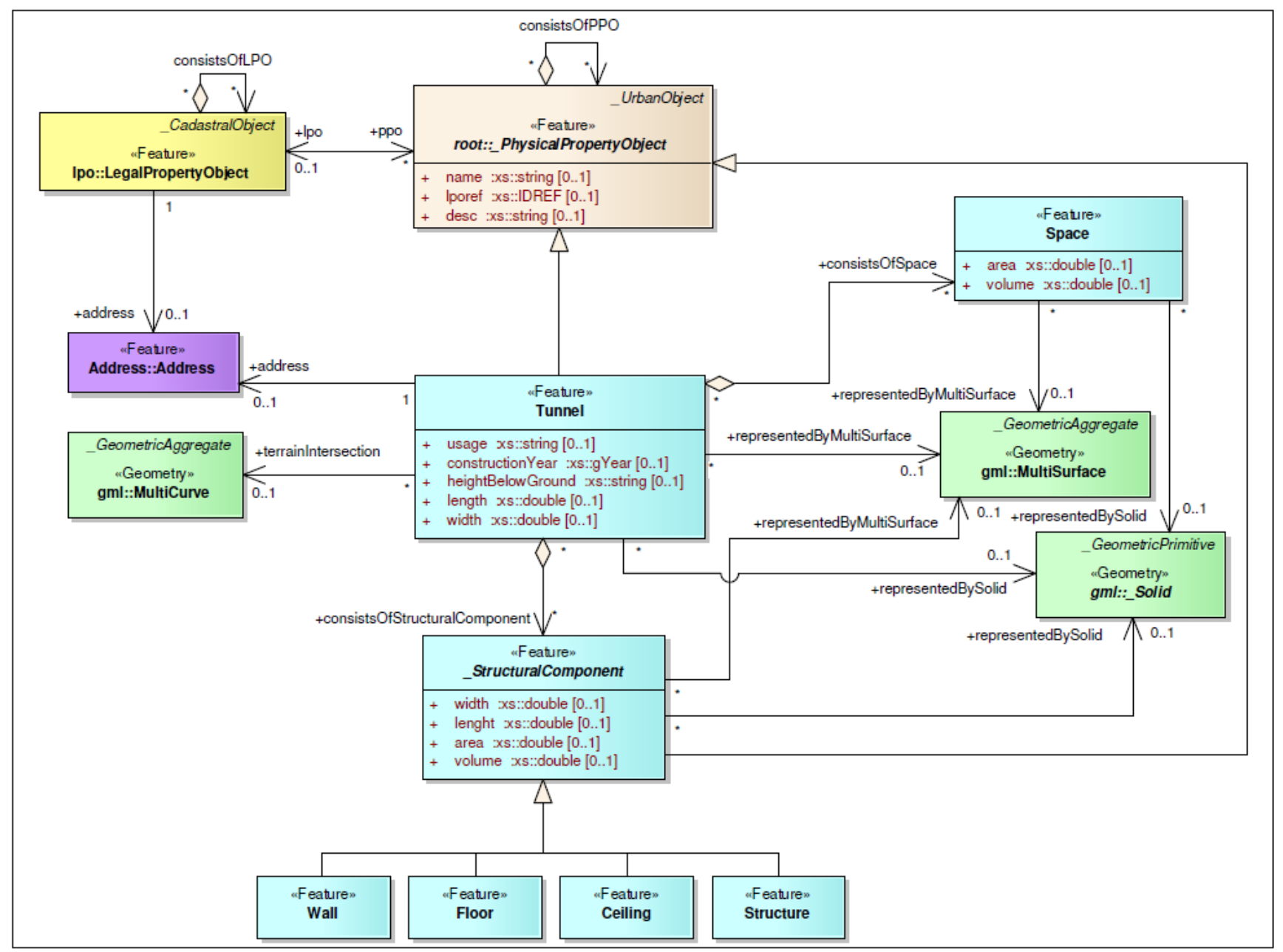

Figure 19. The UML diagram of 3DCDM Tunnel model.

\subsection{DCDM Terrain Model}

Terrain or surface ground is an important part of the physical world. It is also an essential feature for cadastral applications. A combination of terrain and land ownership right objects provides a more realistic representation of the legal world. 2.5D cadastres, representations of land parcels on a 3D ground surface, are a long term practice in the land administration domain [30,31]. 3D cadastres, a combination of 3D parcels with the terrain model, will represent more realistic legal models and facilities to better understand the location and boundaries of land ownership objects, especially when they are located 
below the ground surface. The $3 D C D M$ supports the terrain model. Figure 23 illustrates the UML diagram of the terrain model in the $3 D C D M$.

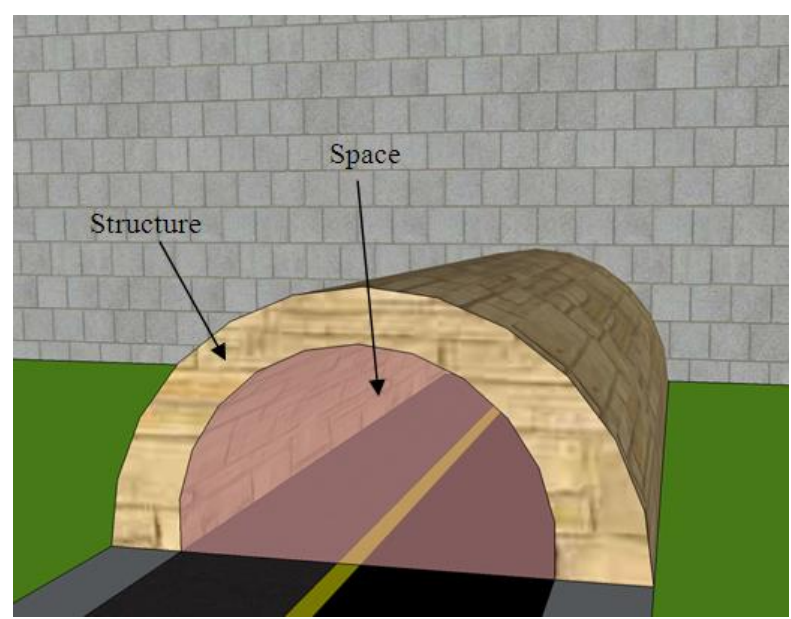

Figure 20. Example of a tunnel model consists of structural components and a space.

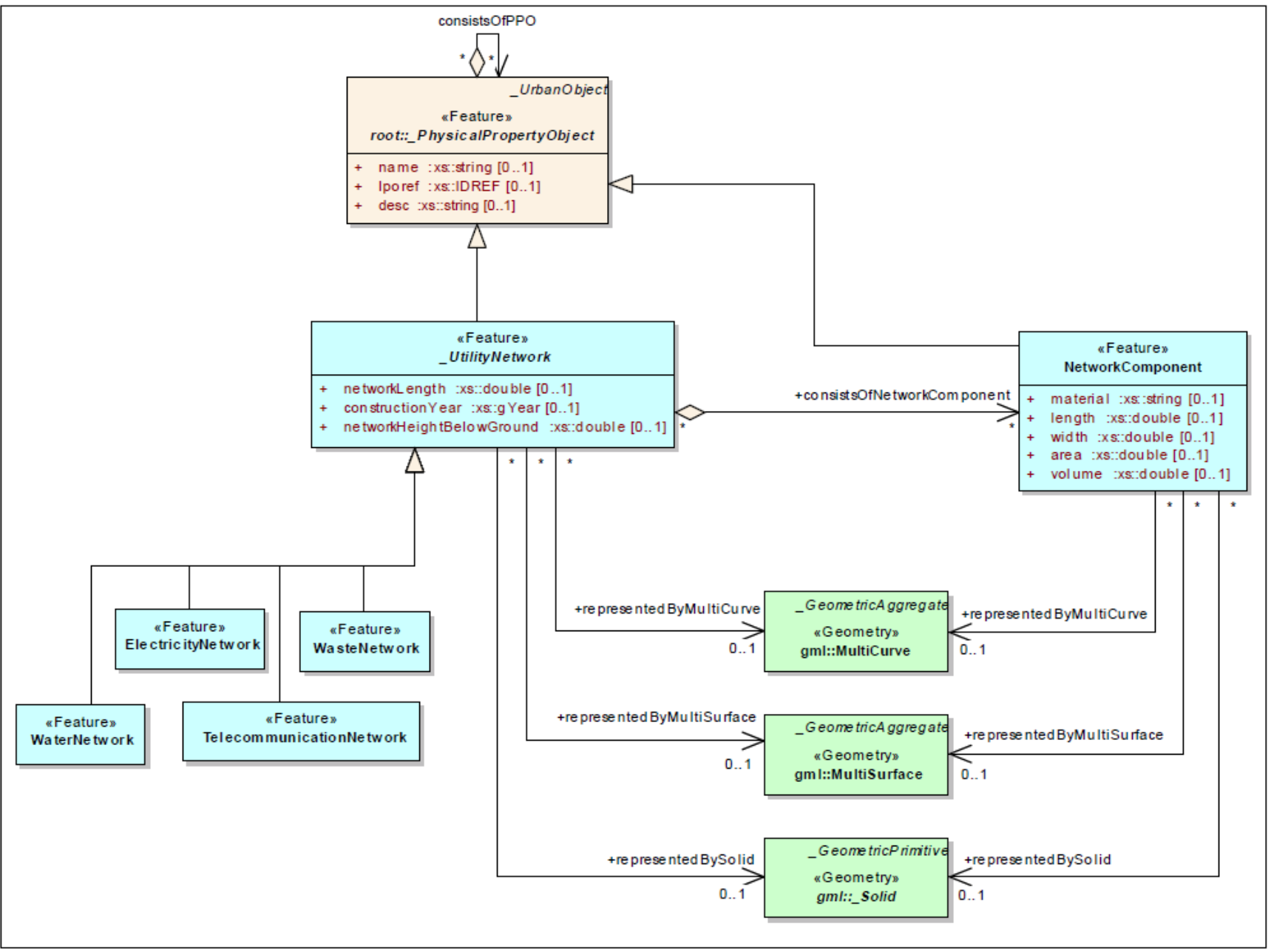

Figure 21. The UML diagram of 3DCDM UtilityNetwork model. 


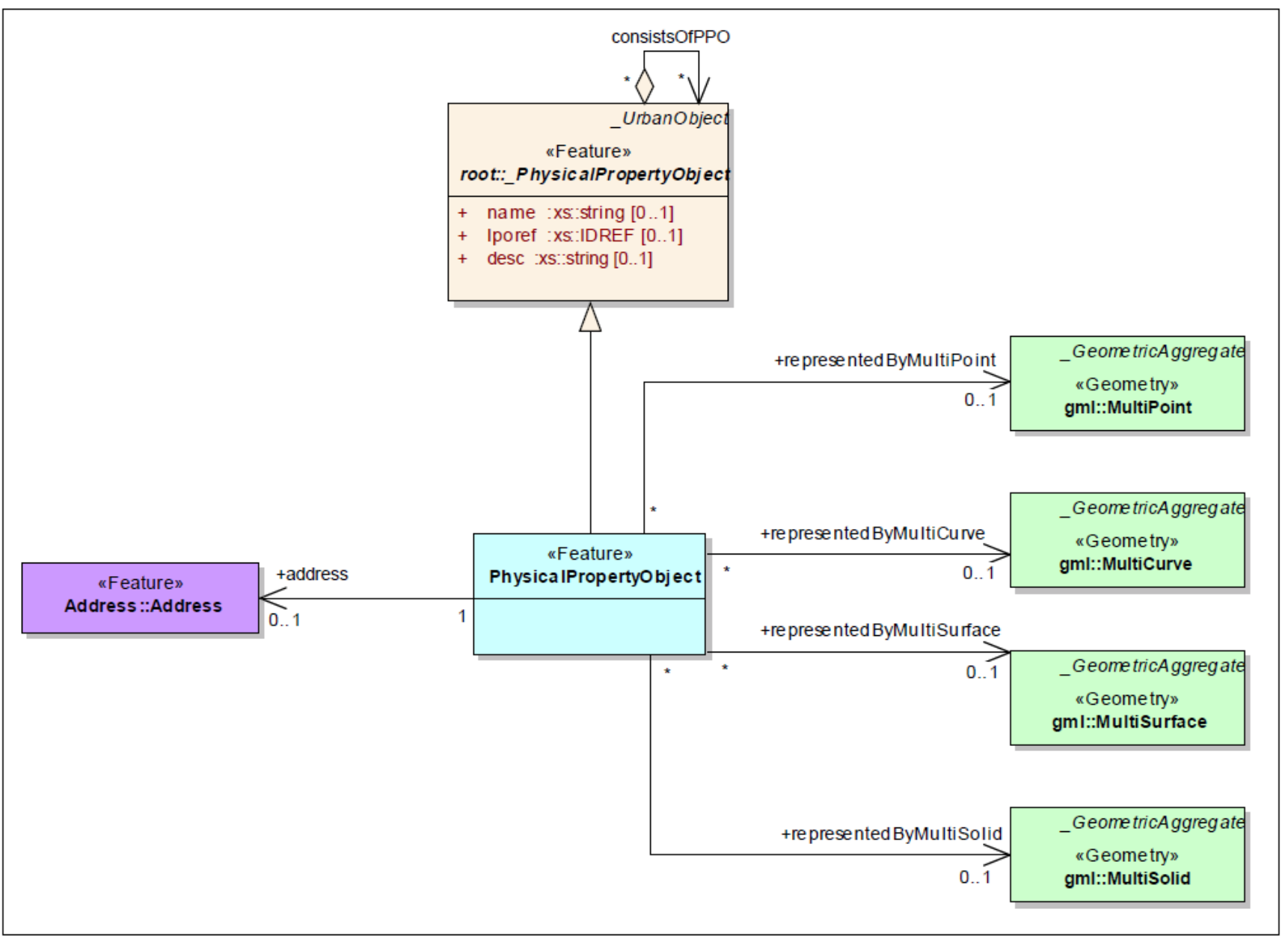

Figure 22. The UML diagram of 3DCDM PhysicalPropertyObject model.

Terrain

In the $3 D C D M$, the terrain is represented by the class Terrain. Terrain is subclasses of Abstract class _UrbanObject (Figure 23).

TerrainSource

Terrain consists of one or more entities of class TerrainSource. TerrainSource are subclasses of Abstract class_UrbanObject (Figure 23).

TIN

In $3 D C D M$, the terrain can be represented as a Triangulated Irregular Network (TIN). TIN is created using GML geometry class gml:Tin (Figure 23).

DEM

The terrain can be represented as a multiple 3D points or DEM in the $3 D C D M$ model. $D E M$ is created using GML geometry class gml:MultiPoint (Figure 23). 


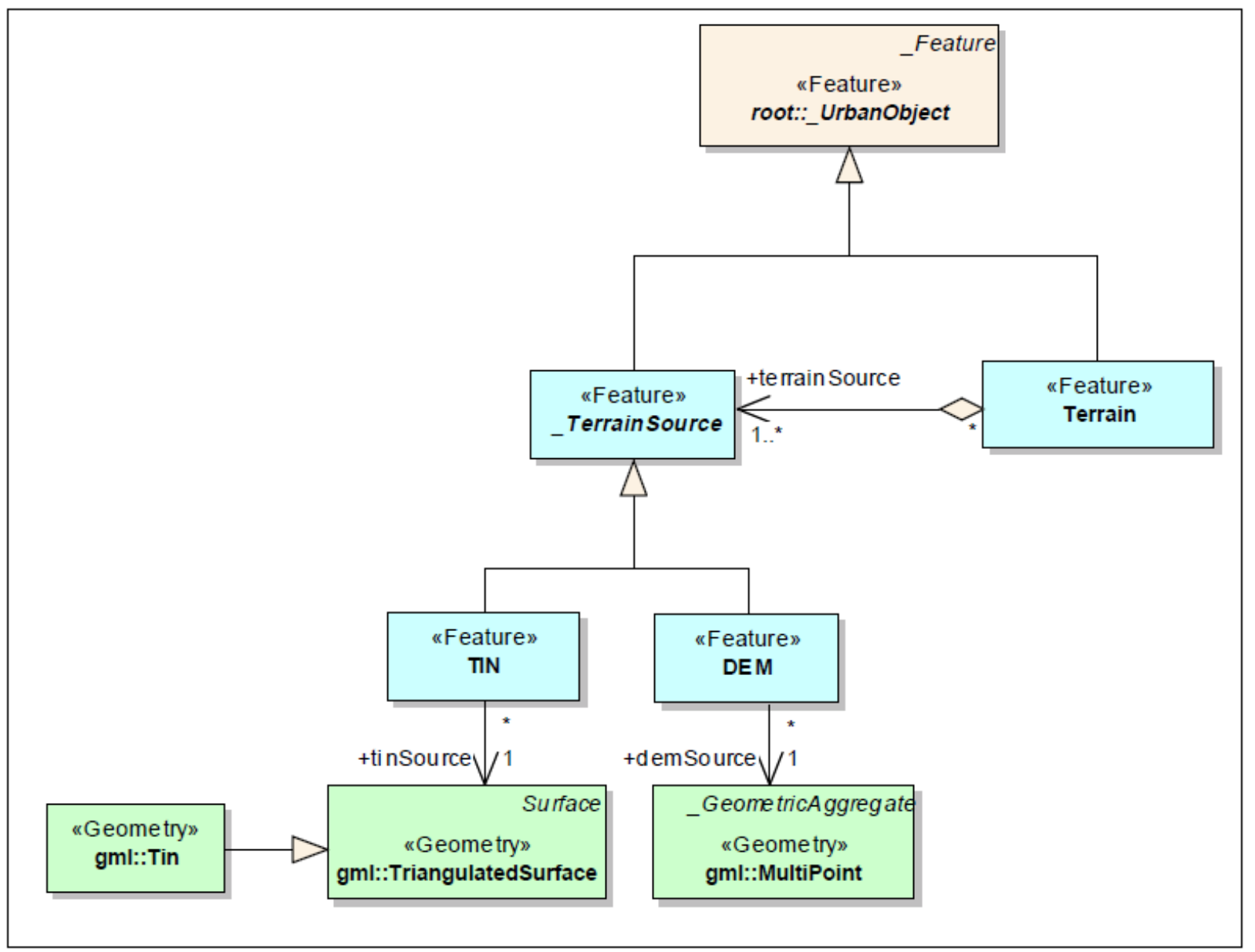

Figure 23. The UML diagram of 3DCDM terrain model.

\begin{tabular}{|ll|}
\hline \multicolumn{1}{|c|}{$\begin{array}{c}\text { «codelist» } \\
\text { rrrType }\end{array}$} \\
\hline+ & ownership \\
+ & common Own ership \\
+ & easement \\
+ & covenant \\
+ & license \\
\hline
\end{tabular}

\begin{tabular}{|ll|}
\hline & \multicolumn{1}{|c|}{ Ipodelist» } \\
\hline+ & lot \\
+ & easement \\
+ & common Property \\
+ & reserve \\
+ & airSpace \\
\hline
\end{tabular}
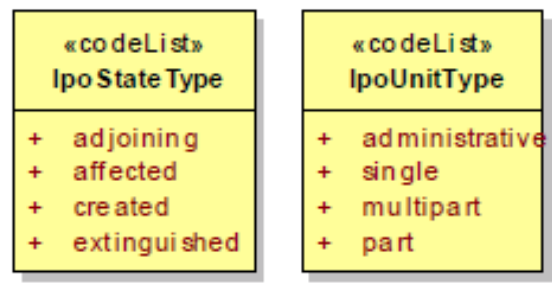

\begin{tabular}{|l|l|}
\hline \multicolumn{1}{|c|}{$\begin{array}{c}\text { acodelist» } \\
\text { landUse Type }\end{array}$} \\
\hline+ & residential \\
+ & industry \\
+ & mixedUse \\
+ & mining \\
+ & agriculture \\
+ & road \\
+ & sea \\
\hline
\end{tabular}

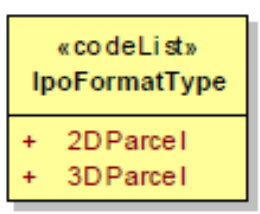

\begin{tabular}{|c|}
\hline $\begin{array}{l}\text { \& co deList» } \\
\text { surFormatType }\end{array}$ \\
\hline $\begin{array}{l}\text { + standard } \\
+ \text { building } \\
+ \text { strata }\end{array}$ \\
\hline
\end{tabular}

\begin{tabular}{|ll|}
\hline \multicolumn{1}{|c|}{$\begin{array}{c}\text { \& codeList» } \\
\text { purposeType }\end{array}$} \\
\hline+ & normal \\
+ & traverse \\
+ & sideshot \\
+ & topo \\
\hline
\end{tabular}
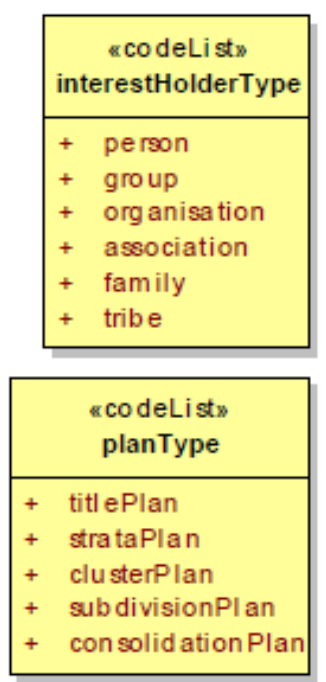

acodelist. landUse Type

+ agriculture

+ ind ustry

+ mining

+ mixedUse

+ residential

+ road

+ sea

\begin{tabular}{|l|}
\hline $\begin{array}{c}\text { ecodelist» } \\
\text { monumentType }\end{array}$ \\
\hline+ peg \\
+ pin \\
+ nail \\
\hline
\end{tabular}

* co de List: pointStateType

+ existing

Figure 24. Code lists of the 3DCDM model. 


\subsection{Code Lists of the 3DCDM Model}

Code lists are used to describe different enumeration of a data model. They represent the possible examples of the values. They are open and can be extended. Figure 24 shows the code lists used in the 3DCDM model.

\section{Evaluation of the 3DCDM Model}

In order to evaluate the $3 D C D M$ model, the conceptual model quality evaluation framework is used to investigate different aspects of the $3 D C D M$ model. This framework provides certain principles and quality factors which can be used to evaluate the $3 D C D M$ model. These principles and quality factors state that data models should [32]:

- meet the data requirement

- be clear and unambiguous to all (not just the authors)

- be stable in the face of changing data requirements

- be flexible in the face of changing business practices

- be reusable by others

- be consistent with other models covering the same scope (if they were developed following these principles)

- be able to integrate data from different data models.

Quality of models is very important because models represent user requirements and are used as bases for building systems. Model quality is considered from two perspectives; process quality and product quality [33].

For product quality, the focus is on characteristics of the final product. It is used to inspect the problems and deficiencies of the final product. While the process quality does not focus on the final product, its focus is on the process which is used to build the product [34].

An international standard has now been established for evaluating the quality of software products. However there is no equivalent standard for evaluating the quality of conceptual models. While a range of quality frameworks have been proposed in the literature, none of these have been widely accepted in practice and none has emerged as a potential standard [32].

Various quality evaluation frameworks have been developed such as [35-39]. Among them, Moody [40] developed a conceptual model quality evaluation framework which highlights eight quality factors: completeness, integrity, flexibility, understandability, correctness, simplicity, integration, and implementability. Every factor has a detailed metric for its evaluation. This framework was applied to ensure process quality in this research. Various review meetings were held during the model development stage.

The definitions of the quality factors are [40]:

Correctness was defined as whether the model conforms to the rules of the data modelling technique (i.e., whether it is a valid data model). This includes diagramming conventions, naming rules, definition rules, rules of composition, and normalisation.

Completeness refers to whether the data model contains all information required to support the required functionality of the system. 
Integrity is defined as whether the data model defines all business rules that apply to the data.

Simplicity means that the data model contains the minimum possible entities and relationships.

Flexibility is defined as the ease with which the data model can cope with business and/or regulatory change.

Integration is defined as the consistency of the data model with the rest of the organisation's data.

Understandability is defined as the ease with which the concepts and structures in the data model can be understood.

Implementability is defined as the ease with which the data model can be implemented within the time, budget, and technology constraints of the project.

Table 1. The 3DCDM model quality factors.

\begin{tabular}{|c|c|c|}
\hline Quality Factor & Description & $\begin{array}{l}\text { Rate }(1=\text { poor; } \\
5=\text { excellent })\end{array}$ \\
\hline Correctness & $\begin{array}{l}\text { The } 3 D C D M \text { model was developed based on the ISO/TC standard 19103:2006 (Geographic } \\
\text { information-Conceptual schema language) and conforms with its technical specifications. } \\
\text { The } 3 D C D M \text { model follows diagramming conventions, naming rules, and all other necessary conditions. } \\
\text { However, the } 3 D C D M \text { model requires inspection by a professional data modeller. }\end{array}$ & 3 \\
\hline Completeness & $\begin{array}{l}\text { The } 3 D C D M \text { model was developed based on the business analysis. In this stage, the model supports most } \\
\text { of the user requirements of the } 3 \mathrm{D} \text { cadastres. Requirements are changing. Therefore, it is essential to } \\
\text { update the user requirements after developing the } 3 D C D M \text { model. }\end{array}$ & 4 \\
\hline Integrity & $\begin{array}{l}\text { In this stage, the } 3 D C D M \text { model defines limited business rules that apply to the data. The } 3 D C D M \text { model } \\
\text { should be assessed by the users to identify how well the model defines the business rules. }\end{array}$ & 3 \\
\hline Simplicity & $\begin{array}{l}\text { The } 3 D C D M \text { model contains the minimum possible entities and relationship mostly because it utilises the } \\
\text { concept of Legal Property Object. However, the } 3 D C D M \text { model requires inspection by a professional } \\
\text { data modeller. }\end{array}$ & 4 \\
\hline Flexibility & $\begin{array}{l}\text { Fully functioning } 3 \mathrm{D} \text { cadastres are yet to be implemented the world. This model can facilitate the } \\
\text { implementation of the } 3 \mathrm{D} \text { cadastre. Therefore, it is not possible to truly discuss how the model } \\
\text { would cope with business changes. However the } 3 D C D M \text { model was developed based on the } \\
\text { idea that } 3 \mathrm{D} \text { cadastres should be developed in such a way that it can help other applications, } \\
\text { such as property management and urban planning. }\end{array}$ & 4 \\
\hline
\end{tabular}

Integration

The $3 D C D M$ model was developed for managing stratified land ownership rights. On the one hand, the $3 D C D M$ model requires $3 \mathrm{D}$ data $(\mathrm{X}, \mathrm{Y}, \mathrm{Z})$ to be able to create and represent the $3 \mathrm{D}$ models. On the other hand, architectural or engineering plans are required to create the physical object on the data model.

However, most of the jurisdictions maintain limited 3D data or physical information. 


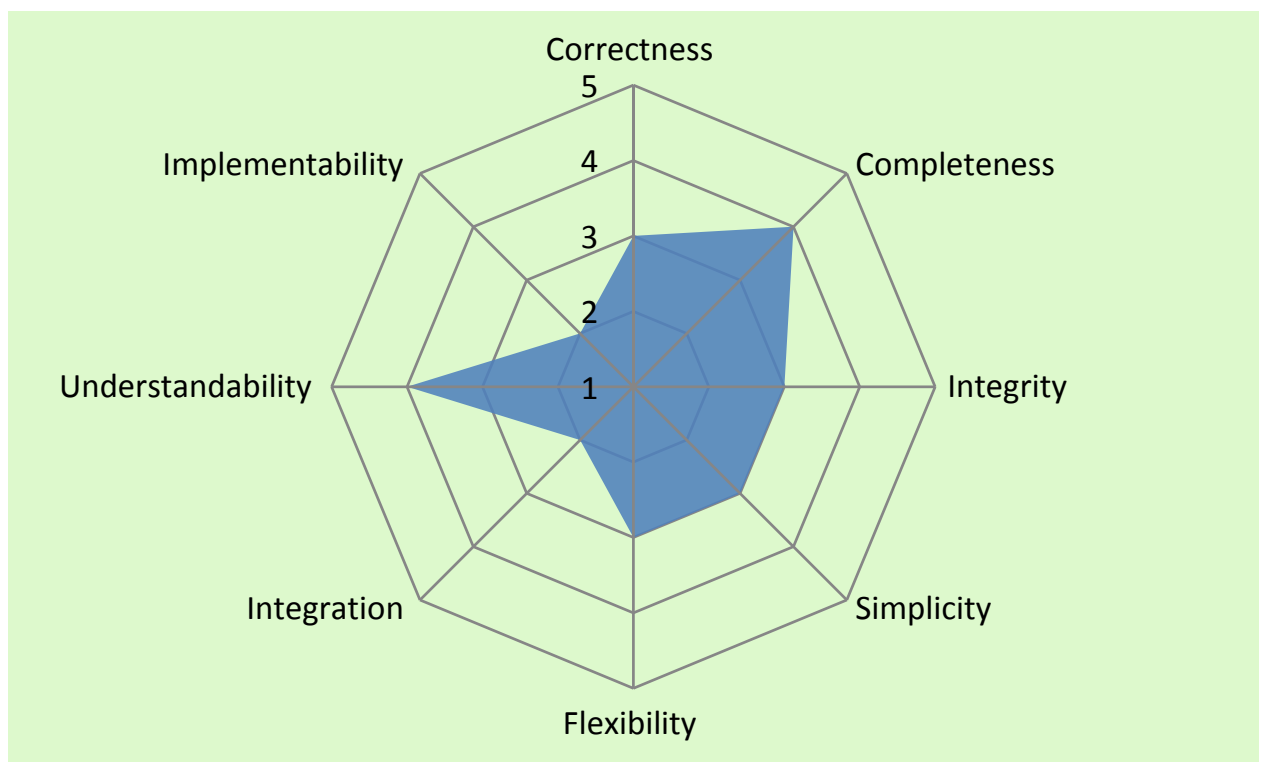

Figure 25. Subjective ratings of the 3DCDM model quality.

In this framework, each quality factor is rated using a $1-5$ scale $(1=$ poor; $5=$ excellent $)$, which was used by the model reviewers. The result of the overall subjective ratings can be presented in the form of Radar or Kiviat charts.

The $3 D C D M$ was validated using a case study approach, which enabled the definition of quality factors. The $3 D C D M$ was rated based on these factors by the project's stakeholders and the results are listed in Table 1. The overall rating of the 3DCDM model is 4 . This is a satisfactory rating given the current lack of available and appropriate 3D data. However, it is likely that, with subsequent use, implementation, and relevant feedback the correctness value will improve over time. In the case study, a two-storey building was used to consider relevant legal (ownership boundaries, ownership information, common properties, and an easement) and physical information (the width, length, and height of walls, roofs, and ceilings). An arbitrary coordinate system was used to extract all coordinates of the required points. From these points various properties, such as width and length of the walls and units, were determined. This information was then inserted into the appropriate XML schemas such as Building, LegalpropertyObject, InterestHolder, Survey, CadastralPoints, and Terrain to demonstrate the validity of the $3 D C D M$ to model and produce the $3 \mathrm{D}$ legal objects of the building and their physical counterparts. The result of this exercise showed that all 3D legal objects of the building and their corresponding physical objects can be effectively modelled using the $3 D C D M$ model.

Figure 25 illustrates the result of the overall subjective ratings of the $3 D C D M$ model, which is represented in a Radar chart.

The subjective ratings shown in Figure 25 clearly indicate what the strengths and weaknesses are in the model. Ratings below 3 are considered "poor" and ratings above 3 are "good". This provides feedback to the analyst as to where the model needs to be improved. Subjective quality ratings are useful for improving the quality of the model, choosing between alternatives and comparison over time improves processes. 


\section{Process of Changes in the 3DCDM}

Above-presented conceptual and logical models of the 3DCDM model have been developed based on the business requirements collected from the project's stakeholders. Of course, these requirements do not cover the needs of all jurisdictions in the world. Hence, changes in the 3DCDM model are required to meet the requirements of a jurisdiction [23].

Based on the level of changes, both conceptual and logical models of the 3DCDM model can be modified. The modification in conceptual model happens in the package level (Figure 8) where a jurisdiction, for example needs, to add a new package in the model. There should be obviously correct associations between added and existing packages in the conceptual model. There is no need to delete a package from the conceptual model if the package is not usable, since the packaging model (modular design) allows users of the data model to avoid utilising unnecessary packages.

Modification in the logical model happens at both the attribute and class levels (Figures 12-19 and 21-23) where a jurisdiction would like to add a new attribute in an existing class or add a new class into the model. Adding attributes is not a complex process according to data modelling rules. However, adding new classes requires one to follow certain data modelling rules to ensure integrity in all components of the data model.

\section{Conclusions}

Integration of legal and physical objects in virtual 3D city models and cadastral data models increases the usability of the data models. They can support various applications such as land development processes and 3D cadastres that require both legal and physical information. Few researchers investigated to extend CityGML and BIM to support legal objects; however, no cadastral data models extended to fully support physical objects.

This paper proposes the $3 D C D M$ model that is developed based on the core cadastral data models and extended to support urban physical objects. The conceptual and logical models of the 3DCDM are presented in detail. The aim is to explore all elements and components of the 3DCDM model, including its classes, attributes, and associations.

For this purpose, the package view of 3DCDM was presented at the first step to provide the overall view of the 3DCDM's components. They are separated into two hierarchies in the 3DCDM model: legal and physical.

Then, 12 sub-models or modules of the 3DCDM were presented, including 3DCDM Geometry Model, 3DCDM Root Model, 3DCDM LegalPropertyObject Model, 3DCDM InterestHolder Model, 3DCDM Survey Model, 3DCDM CadastralPoints Model, 3DCDM Building Model, 3DCDM Land Model, 3DCDM Tunnel Model, 3DCDM UtilityNetwork Model, 3DCDM PhysicalPropertyObject Model, and 3DCDM Terrain Model.

Sub-models are selected based on the user requirements and the application. For example, if the purpose of using the 3DCDM is to model a building, only 3DCDM Building Model and 3DCDM Root Model are used. The 3DCDM Root Model must be used in each implementation of the 3DCDM model. Since each jurisdiction has different cadastral requirements, the data model should be modified to meet those requirements. 
The 3DCDM was validated by the project's stakeholders. However, further research is required to validate the model and examine approaches to implementation. Also, modelling legal objects in different LODs needs more investigation. The logical model of the 3DCDM is utilised as a base to develop the physical model of the $3 D C D M$.

\section{Acknowledgments}

The authors wish to acknowledge the support of the Australian Research Council Linkage Project (LP110200178) on Land and Property information in 3D, its industry partners and the members of the Centre for Spatial Data Infrastructures and Land Administration in the Department of Infrastructure Engineering, The University of Melbourne, in the preparation of this paper and the associated research. However, the views expressed in the paper are those of the authors and do not necessarily reflect those of these groups.

\section{Author Contributions}

All four authors have contributed to the work presented in this paper. From 2010, all have investigated different aspects of the ARC-Linkage project "Land and Property information in 3D". Abbas Rajabifard and Mohsen Kalantari supervised the project and worked on the development of a road map for the project. Davood Shojaei worked on the development of requirements for 3D visualisation systems. Ali Aien worked on 3D cadastral data modelling. All four authors contributed to the writing.

\section{Conflicts of Interest}

The authors declare no conflict of interest.

\section{References}

1. OGC. OGC City Geography Markup Language (CityGML) Encoding Standard; Open Geospatial Consortium Inc.: Wayland, MA, USA, 2012.

2. ISO. ISO 19152:2012. International Standard, Geographic Information-Land Administration Domain Model (LADM); ISO: Geneva, Switzerland, 2012.

3. ePlan. ePlan Model Version 1.0; Intergovernmental Committee on Surveying and Mapping: Sydney, Australia, 2010; p 61.

4. Aien, A.; Kalantari, M.; Rajabifard, A.; Williamson, I.; Wallace, J. Towards integration of 3D legal and physical objects in cadastral data models. Land Use Policy 2013, 35, 140-154.

5. Dsilva, M.G. Feasibility Study on CityGML for Cadastral Purposes. Master's Thesis, Eindhoven University of Technology, Eindhoven, The Netherlands, 2009.

6. El-Mekawy, M.; Östman, A. Feasibility of Building Information Models for 3D Cadastre in Unified City Models. Int. J. E-Plan. Res. (IJEPR). 2012, 1, 35-58.

7. El-Mekawy, M.; Östman, A.; Shahzad, K. Towards interoperating CityGML and IFC building models: A unified model based approach. In Advances in 3D Geo-Information Sciences; Kolbe, T.H., König, G., Nagel, C., Eds.; Springer: Berlin, Heidelberg, Germany, 2011; pp. 73-93.

8. Çă̆daş, V. An application domain extension to CityGML for immovable property taxation: A Turkish case study. Int. J. Appl. Earth Obs. Geoinf. 2013, 21, 545-555. 
9. van den Brink, L.; Stoter, J.E.; Zlatanova, S. Establishing a national standard for 3D topographic data compliant to CityGML. Int. J. Geogr. Inf. Sci. 2013, 27, 92-113.

10. Gozdz, K.; Pachelski, W.; Van Oosterom, P.J.M.; Coors, V. The possibilities of using CityGML for 3D representation of buildings in the cadastre. In Proceedings of 4th International Workshop on 3D Cadastres, Dubai, UAE, 9-11 November 2014; pp. 339-361.

11. Ying, S.; Jin, F.; Guo, R.; Li, L.; Yang, J.; Zhou, Y. The conversion from CityGML to 3D property units. In Proceedings of 4th International Workshop on 3D Cadastres, Dubai, UAE, 9-11 November 2014; pp. 109-121.

12. El-Mekawy, M.; Paasch, J.; Paulsson, J. Integration of 3D cadastre, 3D property formation and BIM in Sweden. In Proceedings of 4th International Workshop on 3D Cadastres, Dubai, UAE, 9-11 November 2014; pp. 17-34.

13. Isikdag, U.; Horhammer, M.; Zlatanova, S.; Kathmann, R.; Van Oosterom, P.J.M. Semantically rich 3D building and cadastral models for valuation. In Proceedings of 4th International Workshop on 3D Cadastres, Dubai, UAE, 9-11 November 2014; pp. 35-53.

14. Soon, K.H.; Thompson, R.; Khoo, V. Semantics-based fusion for CityGML and 3D LandXML. In Proceedings of 4th International Workshop on 3D Cadastres, Dubai, UAE, 9-11 November 2014; pp. 323-338.

15. Rönsdorf, C.; Wilson, D.; Stoter, J. Integration of land administration domain model with CityGML for 3D cadastre. In Proceedings of 4th International Workshop on 3D Cadastres, Dubai, UAE, 9-11 November 2014; pp. 313-322.

16. OGC. The OGC LandInfra Conceptual Model; Open Geospatial Consortium Inc.: Wayland, MA, USA, 2014.

17. Henssen, J. Basic principles of the main cadastral systems in the world. In Proceedings of Seminar Modern Cadastres and Cadastral Innovations, Delft, The Netherlands, 16 May 1995; The International Federation of Surveyors (FIG): Delft, The Netherlands, 1995; pp. 5-12.

18. Van Oosterom, P.; Lemmen, C.; Ingvarsson, T.; van der Molen, P.; Ploeger, H.; Quak, W.; Stoter, J.E.; Zevenbergen, J. The core cadastral domain model. Comput. Environ. Urban Sys. 2006, $30,627-660$.

19. Kaufmann, J.; Steudler, D. Cadastre 2014: A vision for future cadastral system. In Proceedings of FIG XXI International Congress, Brighton, UK, 19-25 July 1998.

20. von Meyer, N. GIS and Land Records: The ArcGIS Parcel Data Model; ESRI Press: Redlands, CA, USA, 2004; p. 150.

21. Steudler, D. Swiss cadastral core data model—Experiences of the last 15 years. Comput. Environ. Urban Sys. 2005, 30, 600-613.

22. FGDC. Cadastral Data Content Standard for the National Spatial Data Infrastructure-Version 1.4; Subcommittee on Cadastral Data, Federal Geographic Data Committee: Reston, VA, USA, 2008; p. 108.

23. Kalantari, M.; Rajabifard, A.; Wallace, J.; Williamson, I.P. Spatially referenced legal property objects. Land Use Policy 2008, 25, 173-183.

24. Lemmen, C. A Domain Model for Land Administration. PhD Thesis, Delft University of Technology, Delft, The Netherlands, 2012. 
25. Kalantari, M. Cadastral Data Modelling-A Tool for e-Land Administration; The University of Melbourne: Melbourne, Australia, 2008.

26. Aien, A.; Kalantari, M.; Rajabifard, A.; Williamson, I.; Bennett, R. Utilising data modelling to understand the structure of 3D cadastres. J. Spat. Sci. 2013, 58, 215-234.

27. ISO19107. Geographic Information-Spatial Schema; AS/NZS ISO: Geneva, Switzerland, 2005; p. 171.

28. Foley, J.D.; van Dam, A.; Feiner, S.K.; Hughes, J.F. Computer Graphics: Principles and Practice, 2nd ed.; Addison-Wesley: Boston, MA, USA, 1995; p. 699.

29. ISO19136. Geographic information-Geography Markup Language (GML); ISO: Geneva, Switzerland, 2007; p. 394.

30. Bydlosz, J. The 3D cadastre aspects in international standards and solutions. In Proceedings of FIG Commission 3 Workshop 2012, Spatial Information, Informal Development, Property and Housing, Athens, Greece, 10-14 December 2012; FIG: Athens, Greece, 2012; p. 10.

31. Stoter, J.; van Oosterom, P.J.M.; Ploeger, H. D.; Aalders, H. Conceptual 3D cadastral model applied in several countries. In Proceedings of FIG Working Week 2004, Appropriate Technologies for Good Land Administration II-3D Cadastre, Athens, Greece, 22-27 May 2004; FIG: Athens, Greece, 2004; p. 27.

32. West, M. Developing High Quality Data Models; Morgan Kaufmann: Burlington, MA, USA, 2011; p. 408.

33. Moody, D.L. Metrics for Evaluating the Quality of Entity Relationship Models. Lect. Note. Comput. Sci. 1998, 1507, 211-225.

34. Alkhaldi, N. Participatory quality management of ontologies in enterprise modelling. In Proceedings of CAiSE 2012 Doctoral Consortium, Gdansk, Poland, 26 June 2012; p. 12.

35. Assenova, P.; Johannesson, P. Improving quality of conceptual modelling by the use of scheme transformation. In Proceedings of the 15th International Conference of Conceptual Modelling (ER'96), Cottbus, Germany, 7-10 October 1996; pp. 277-291.

36. Kesh, S. Evaluating the quality of entity relationship models. Inf. and Softw. Technol. 1995, 37, 681-689.

37. Moody, D.L.; Shanks, G. What makes a good data model? A framework for evaluating and improving the quality of entity relationship models. Aust. Comput. J. 1998, 30, 97-110.

38. Poels, G.; Dedene, G. Measures of assessing dynamic complexity aspects of objectoriented conceptual schemes. In Proceedings of the 9th International Conference on Conceptual Modelling, ER'2000, Salt Lake City, UT, USA, 9-12 October 2000; pp. 513-526

39. Shanks, G. Conceptual Data Modelling: an empirical study of expert and novice data modellers. Australas. J. Inf. Sys. 1997, 4, 63-73.

40. Moody, D.L. Measuring the quality of data models: An empirical evaluation of the use of quality metrics in practice. In Proceedings of the 11th European Conference on Information Systems, ECIS 2003, Naples, Italy, 16-21 June 2003; pp. 1337-1352.

(C) 2015 by the authors; licensee MDPI, Basel, Switzerland. This article is an open access article distributed under the terms and conditions of the Creative Commons Attribution license (http://creativecommons.org/licenses/by/4.0/). 